Supporting Information for

\title{
Theoretical Insights into Superior Nitrate Reduction to Ammonia Performance of Copper Catalysts
}

Tao Hu, ${ }^{\dagger}$ Changhong Wang, ${ }^{\dagger}$ Mengting Wang, ${ }^{\dagger}$ Chang Ming Li,,${ }^{\prime \prime}$ and Chunxian Guo, ${ }^{\prime}$ *

†nstitute of Materials Science and Devices, School of Materials Science and Engineering, Suzhou

University of Science and Technology, Suzhou 215009, China

Institute for Cross-field Science and College of Life Science, Qingdao University, Qingdao 200671, China

*E-mail: cxguo@usts.edu.cn

\section{Supplementary summary}

Elementary steps consideration

Thermodyamic data

Minimum energy path and activation barriers

Adsorption configuration of $* \mathrm{NO}$ and $* \mathrm{NO}_{3}$ 


\section{Part I Calculation Details}

Table S1. Elementary steps of NRA1

\begin{tabular}{|c|c|c|c|}
\hline \multicolumn{4}{|c|}{ NRA1: $\mathrm{NO}_{3}{ }^{-} \rightarrow * \mathrm{NO}_{3}{ }^{-} \rightarrow * \mathrm{NO}_{2} \rightarrow * \mathrm{NO} \rightarrow * \mathrm{~N} \rightarrow * \mathrm{NH} \rightarrow * \mathrm{NH}_{2} \rightarrow * \mathrm{NH}_{3} \rightarrow \mathrm{NH}_{3}(\mathrm{~g})$} \\
\hline $\mathrm{NO}_{3}^{-}(1)+* \rightarrow * \mathrm{NO}_{3}+\mathrm{e}^{-}$ & $(r 1)$ & & \\
\hline$* \mathrm{NO}_{3}+2 \mathrm{H}^{+}+2 \mathrm{e}^{-} \rightarrow * \mathrm{NO}_{2}+\mathrm{H}_{2} \mathrm{O}$ & $(r 2)$ & $\begin{array}{l}* \mathrm{NO}_{3} \rightarrow * \mathrm{NO}_{2}+* \mathrm{O} \\
* \mathrm{O}+2 \mathrm{H}^{+}+2 \mathrm{e}^{-} \rightarrow \mathrm{H}_{2} \mathrm{O}\end{array}$ & $\left(r 2^{\prime}\right)$ \\
\hline$* \mathrm{NO}_{2}+2 \mathrm{H}^{+}+2 \mathrm{e}^{-} \rightarrow * \mathrm{NO}+\mathrm{H}_{2} \mathrm{O}$ & $(r 3)$ & $\begin{array}{l}* \mathrm{NO}_{2} \rightarrow * \mathrm{NO}+* \mathrm{O} \\
* \mathrm{O}+2 \mathrm{H}^{+}+2 \mathrm{e}^{-} \rightarrow \mathrm{H}_{2} \mathrm{O}\end{array}$ & $\left(r 3^{\prime}\right)$ \\
\hline$* \mathrm{NO}+2 \mathrm{H}^{+}+2 \mathrm{e}^{-} \rightarrow * \mathrm{~N}+\mathrm{H}_{2} \mathrm{O}$ & $(r 4)$ & $\begin{array}{l}* \mathrm{NO} \rightarrow{ }^{*} \mathrm{~N}+* \mathrm{O} \\
* \mathrm{O}+2 \mathrm{H}^{+}+2 \mathrm{e}^{-} \rightarrow \mathrm{H}_{2} \mathrm{O}\end{array}$ & $\left(r 4^{\prime}\right)$ \\
\hline$* \mathrm{~N}+\mathrm{H}^{+}+\mathrm{e}^{-} \rightarrow * \mathrm{NH}$ & $(r 5)$ & $* \mathrm{~N}+* \mathrm{H} \rightarrow * \mathrm{NH}$ & $\left(r 5^{\prime}\right)$ \\
\hline$* \mathrm{NH}+\mathrm{H}^{+}+\mathrm{e}^{-} \rightarrow * \mathrm{NH}_{2}$ & $(r 6)$ & $* \mathrm{NH}+* \mathrm{H} \rightarrow * \mathrm{NH}_{2}$ & $\left(r 6{ }^{\prime}\right)$ \\
\hline$* \mathrm{NH}_{2}+\mathrm{H}^{+}+\mathrm{e}^{-} \rightarrow * \mathrm{NH}_{3}$ & $(r 7)$ & $* \mathrm{NH}_{2}+* \mathrm{H} \rightarrow * \mathrm{NH}_{3}$ & $\left(r 7^{\prime}\right)$ \\
\hline$* \mathrm{NH}_{3} \rightarrow *+\mathrm{NH}_{3}(\mathrm{~g})$ & $(r 8)$ & & \\
\hline $\mathrm{NO}_{3}^{-}+9 \mathrm{H}^{+}+8 \mathrm{e}^{-} \rightarrow \mathrm{NH}_{3}+6 \mathrm{H}_{2} \mathrm{O}$ & (total) & & \\
\hline
\end{tabular}

Eqations $r 1, r 2, r 3, r 4, r 5, r 6, r 7$, and $r 8$ were used for thermodynamics calculation. Equations $r 2$ ', $r 3^{\prime}, r 4^{\prime}, r 5^{\prime}, r 6^{\prime}$, and $r 7^{\prime}$ were used to calculate the kinetics of deoxygenation process of $* \mathrm{NO}_{x}$ and hydrogenation of $* \mathrm{NH}_{y}$.

Correction of $\mathrm{pH}$ to Gibbs free energy':

$\Delta \mathrm{G}_{1}=\Delta \mathrm{G}_{1}{ }^{0}$

$\Delta \mathrm{G}_{2}=\Delta \mathrm{G}_{2}{ }^{0}+2 k_{\mathrm{B}} \mathrm{T} \ln 10 \times \mathrm{pH}$

$\Delta \mathrm{G}_{3}=\Delta \mathrm{G}_{3}{ }^{0}+2 k_{\mathrm{B}} \mathrm{T} \ln 10 \times \mathrm{pH}$

$\Delta \mathrm{G}_{4}=\Delta \mathrm{G}_{4}{ }^{0}+2 k_{\mathrm{B}} \mathrm{T} \ln 10 \times \mathrm{pH}$

$\Delta \mathrm{G}_{5}=\Delta \mathrm{G}_{5}^{0}+k_{\mathrm{B}} \mathrm{T} \ln 10 \times \mathrm{pH}$

$\Delta \mathrm{G}_{6}=\Delta \mathrm{G}_{6}{ }^{0}+k_{\mathrm{B}} \mathrm{T} \ln 10 \times \mathrm{pH}$

$\Delta \mathrm{G}_{7}=\Delta \mathrm{G}_{7}{ }^{0}+k_{\mathrm{B}} \mathrm{Tln} 10 \times \mathrm{pH}$

$\Delta \mathrm{G}_{8}=\Delta \mathrm{G}_{8}{ }^{0}$ 
Table S2. Elementary steps of NRA2

\begin{tabular}{|c|c|c|c|}
\hline \multicolumn{4}{|c|}{ NRA2: $\mathrm{NO}_{3}{ }^{-} \rightarrow * \mathrm{NO}_{3}{ }^{-} \rightarrow * \mathrm{NO}_{3} \rightarrow * \mathrm{NO}_{2} \rightarrow * \mathrm{NO} \rightarrow * \mathrm{NOH} \rightarrow * \mathrm{NHOH} \rightarrow * \mathrm{NH}_{2} \mathrm{OH} \rightarrow * \mathrm{NH}_{2} \rightarrow * \mathrm{NH}_{3} \rightarrow \mathrm{NH}_{3}(\mathrm{~g})$} \\
\hline $\mathrm{NO}_{3}^{-}(1)+* \rightarrow * \mathrm{NO}_{3}+\mathrm{e}^{-}$ & $(r 1)$ & & \\
\hline$* \mathrm{NO}_{3}+2 \mathrm{H}^{+}+2 \mathrm{e}^{-} \rightarrow * \mathrm{NO}_{2}+\mathrm{H}_{2} \mathrm{O}$ & $(r 2)$ & $\begin{array}{l}{ }^{*} \mathrm{NO}_{3} \rightarrow * \mathrm{NO}_{2}+* \mathrm{O} \\
* \mathrm{O}+2 \mathrm{H}^{+}+2 \mathrm{e}^{-} \rightarrow \mathrm{H}_{2} \mathrm{O}\end{array}$ & $\left(r 2^{\prime}\right)$ \\
\hline$* \mathrm{NO}_{2}+2 \mathrm{H}^{+}+2 \mathrm{e}^{-} \rightarrow * \mathrm{NO}+\mathrm{H}_{2} \mathrm{O}$ & $(r 3)$ & $\begin{array}{l}* \mathrm{NO}_{2} \rightarrow * \mathrm{NO}+* \mathrm{O} \\
* \mathrm{O}+2 \mathrm{H}^{+}+2 \mathrm{e}^{-} \rightarrow \mathrm{H}_{2} \mathrm{O}\end{array}$ & $\left(r 3^{\prime}\right)$ \\
\hline$* \mathrm{NO}+\mathrm{H}^{+}+\mathrm{e}^{-} \rightarrow * \mathrm{NOH}$ & $(r 9)$ & $* \mathrm{NO}+* \mathrm{H} \rightarrow * \mathrm{NOH}$ & $\left(r 9^{\prime}\right)$ \\
\hline$* \mathrm{NOH}+\mathrm{H}^{+}+\mathrm{e}^{-} \rightarrow * \mathrm{NHOH}$ & $(r 10)$ & $* \mathrm{NOH}+* \mathrm{H} \rightarrow * \mathrm{NHOH}$ & $\left(r 10^{\prime}\right)$ \\
\hline$* \mathrm{NHOH}+\mathrm{H}^{+}+\mathrm{e}^{-} \rightarrow * \mathrm{NH}_{2} \mathrm{OH}$ & $(r 11)$ & $* \mathrm{NHOH}+* \mathrm{H} \rightarrow * \mathrm{NH}_{2} \mathrm{OH}$ & $\left(r 11^{\prime}\right)$ \\
\hline$* \mathrm{NH}_{2} \mathrm{OH}+\mathrm{H}^{+}+\mathrm{e}^{-} \rightarrow * \mathrm{NH}_{2}+\mathrm{H}_{2} \mathrm{O}$ & $(r 12)$ & $* \mathrm{NH}_{2} \mathrm{OH}+* \mathrm{H} \rightarrow * \mathrm{NH}_{2}+\mathrm{H}_{2} \mathrm{O}$ & $\left(r 12^{\prime}\right)$ \\
\hline$* \mathrm{NH}_{2}+\mathrm{H}^{+}+\mathrm{e}^{-} \rightarrow * \mathrm{NH}_{3}$ & $(r 7)$ & $* \mathrm{NH}_{2}+* \mathrm{H} \rightarrow * \mathrm{NH}_{3}$ & $\left(r 7^{\prime}\right)$ \\
\hline$* \mathrm{NH}_{3} \rightarrow *+\mathrm{NH}_{3}(\mathrm{~g})$ & $(r 8)$ & & \\
\hline $\mathrm{NO}_{3}{ }^{-}+9 \mathrm{H}^{+}+8 \mathrm{e}^{-} \rightarrow \mathrm{NH}_{3}+6 \mathrm{H}_{2} \mathrm{O}$ & (total) & & \\
\hline
\end{tabular}

Eqations $r 1, r 2, r 3, r 9, r 10, r 11, r 12, r 7$ and $r 8$ were used for thermodynamics calculation. Equations $r 2^{\prime}, r 3^{\prime}, r 9^{\prime}, r 10^{\prime}, r 11^{\prime}, r 12^{\prime}$, and $r 7^{\prime}$ were used to calculate the kinetics of deoxygenation process of $* \mathrm{NO}_{x}$ and hydrogenation of $* \mathrm{NH}_{y}$.

Correction of $\mathrm{pH}$ to Gibbs free energy

$$
\begin{aligned}
& \Delta \mathrm{G}_{1}=\Delta \mathrm{G}_{1}^{0} \\
& \Delta \mathrm{G}_{2}=\Delta \mathrm{G}_{2}^{0}+2 k_{\mathrm{B}} \mathrm{T} \ln 10 \times \mathrm{pH} \\
& \Delta \mathrm{G}_{3}=\Delta \mathrm{G}_{3}^{0}+2 k_{\mathrm{B}} \mathrm{T} \ln 10 \times \mathrm{pH} \\
& \Delta \mathrm{G}_{9}=\Delta \mathrm{G}_{9}^{0}+k_{\mathrm{B}} \mathrm{T} \ln 10 \times \mathrm{pH} \\
& \Delta \mathrm{G}_{10}=\Delta \mathrm{G}_{10}{ }^{0}+k_{\mathrm{B}} \mathrm{T} \ln 10 \times \mathrm{pH} \\
& \Delta \mathrm{G}_{11}=\Delta \mathrm{G}_{11}{ }^{0}+k_{\mathrm{B}} \mathrm{T} \ln 10 \times \mathrm{pH} \\
& \Delta \mathrm{G}_{12}=\Delta \mathrm{G}_{12}{ }^{0}+k_{\mathrm{B}} \mathrm{T} \ln 10 \times \mathrm{pH} \\
& \Delta \mathrm{G}_{7}=\Delta \mathrm{G}_{7}^{0+} k_{\mathrm{B}} \mathrm{T} \ln 10 \times \mathrm{pH} \\
& \Delta \mathrm{G}_{8}=\Delta \mathrm{G}_{8}{ }^{0}
\end{aligned}
$$


Table S3. Elementary steps of NRA3

\begin{tabular}{|c|c|c|c|}
\hline \multicolumn{4}{|c|}{$\mathrm{NRA} 3: * \rightarrow * \mathrm{NO}_{3} \rightarrow * \mathrm{NO}_{2} \rightarrow * \mathrm{NO} \rightarrow * \mathrm{NOH} \rightarrow * \mathrm{NHOH} \rightarrow * \mathrm{NH} \rightarrow * \mathrm{NH}_{2} \rightarrow * \mathrm{NH}_{3} \rightarrow \mathrm{NH}_{3}(\mathrm{~g})$} \\
\hline $\mathrm{NO}_{3}^{-}(1)+* \rightarrow * \mathrm{NO}_{3}+\mathrm{e}^{-}$ & $(r 1)$ & & \\
\hline$* \mathrm{NO}_{3}+2 \mathrm{H}^{+}+2 \mathrm{e}^{-} \rightarrow * \mathrm{NO}_{2}+\mathrm{H}_{2} \mathrm{O}$ & $(r 2)$ & $\begin{array}{l}{ }^{*} \mathrm{NO}_{3} \rightarrow * \mathrm{NO}_{2}+* \mathrm{O} \\
* \mathrm{O}+2 \mathrm{H}^{+}+2 \mathrm{e}^{-} \rightarrow \mathrm{H}_{2} \mathrm{O}\end{array}$ & $\left(r 2^{\prime}\right)$ \\
\hline$* \mathrm{NO}_{2}+2 \mathrm{H}^{+}+2 \mathrm{e}^{-} \rightarrow * \mathrm{NO}+\mathrm{H}_{2} \mathrm{O}$ & (r3) & $\begin{array}{l}* \mathrm{NO}_{2} \rightarrow * \mathrm{NO}+* \mathrm{O} \\
* \mathrm{O}+2 \mathrm{H}^{+}+2 \mathrm{e}^{-} \rightarrow \mathrm{H}_{2} \mathrm{O}\end{array}$ & $\left(r 3^{\prime}\right)$ \\
\hline$* \mathrm{NO}+\mathrm{H}^{+}+\mathrm{e}^{-} \rightarrow * \mathrm{NOH}$ & $\left(r^{9}\right)$ & $* \mathrm{NO}+* \mathrm{H} \rightarrow * \mathrm{NOH}$ & $\left(r 9^{\prime}\right)$ \\
\hline$* \mathrm{NOH}+\mathrm{H}^{+}+\mathrm{e}^{-} \rightarrow * \mathrm{NHOH}$ & $(r 10)$ & $* \mathrm{NOH}+* \mathrm{H} \rightarrow * \mathrm{NHOH}$ & $\left(r 10^{\prime}\right)$ \\
\hline$* \mathrm{NHOH}+\mathrm{H}^{+}+\mathrm{e}^{-} \rightarrow * \mathrm{NH}+\mathrm{H}_{2} \mathrm{O}$ & $(r 13)$ & $* \mathrm{NHOH}+* \mathrm{H} \rightarrow * \mathrm{NH}+\mathrm{H}_{2} \mathrm{O}$ & $\left(r 13^{\prime}\right)$ \\
\hline$* \mathrm{NH}+\mathrm{H}^{+}+\mathrm{e}^{-} \rightarrow * \mathrm{NH}_{2}$ & $(r 6)$ & $* \mathrm{NH}+* \mathrm{H} \rightarrow * \mathrm{NH}_{2}$ & $\left(r 6^{\prime}\right)$ \\
\hline$* \mathrm{NH}_{2}+\mathrm{H}^{+}+\mathrm{e}^{-} \rightarrow * \mathrm{NH}_{3}$ & $(r 7)$ & $* \mathrm{NH}_{2}+* \mathrm{H} \rightarrow * \mathrm{NH}_{3}$ & $\left(r 7^{\prime}\right)$ \\
\hline$* \mathrm{NH}_{3} \rightarrow *+\mathrm{NH}_{3}(\mathrm{~g})$ & $(r 8)$ & & \\
\hline $\mathrm{NO}_{3}^{-}+9 \mathrm{H}^{+}+8 \mathrm{e}^{-} \rightarrow \mathrm{NH}_{3}+6 \mathrm{H}_{2} \mathrm{O}$ & (total) & & \\
\hline
\end{tabular}

Eqations $r 1, r 2, r 3, r 9, r 10, r 13, r 6, r 7$ and $r 8$ were used for thermodynamics calculation. Equations r2', r3', r9', r10', r13', r6', and $r 7^{\prime}$ were used to calculate the kinetics of deoxygenation process of $* \mathrm{NO}_{x}$ and hydrogenation of $* \mathrm{NH}_{y}$.

Correction of $\mathrm{pH}$ to Gibbs free energy

$\Delta \mathrm{G}_{1}=\Delta \mathrm{G}_{1}^{0}$

$\Delta \mathrm{G}_{2}=\Delta \mathrm{G}_{2}{ }^{0}+2 k_{\mathrm{B}} \mathrm{Tln} 10 \times \mathrm{pH}$

$\Delta \mathrm{G}_{3}=\Delta \mathrm{G}_{3}{ }^{0}+2 k_{\mathrm{B}} \mathrm{Tln} 10 \times \mathrm{pH}$

$\Delta \mathrm{G}_{9}=\Delta \mathrm{G}_{9}{ }^{0}+k_{\mathrm{B}} \mathrm{T} \ln 10 \times \mathrm{pH}$

$\Delta \mathrm{G}_{10}=\Delta \mathrm{G}_{10}{ }^{0}+k_{\mathrm{B}} \mathrm{T} \ln 10 \times \mathrm{pH}$

$\Delta \mathrm{G}_{13}=\Delta \mathrm{G}_{13}{ }^{0}+k_{\mathrm{B}} \mathrm{T} \ln 10 \times \mathrm{pH}$

$\Delta \mathrm{G}_{6}=\Delta \mathrm{G}_{6}{ }^{0}+k_{\mathrm{B}} \mathrm{T} \ln 10 \times \mathrm{pH}$

$\Delta \mathrm{G}_{7}=\Delta \mathrm{G}_{7}^{0}+k_{\mathrm{B}} \mathrm{T} \ln 10 \times \mathrm{pH}$

$\Delta \mathrm{G}_{8}=\Delta \mathrm{G}_{8}{ }^{0}$ 
In the senarios of neutral and alkaline solution, the $\mathrm{H}^{+}$for NRA comes from $\mathrm{H}_{2} \mathrm{O}$, and the equations can be written as the following equations.

Table S4. NRA1 reaction steps in acidic solution and alkaline solution

\begin{tabular}{|c|c|c|c|}
\hline \multicolumn{4}{|c|}{ NRA1: $\mathrm{NO}_{3}{ }^{-} \rightarrow * \mathrm{NO}_{3}{ }^{-} \rightarrow * \mathrm{NO}_{2} \rightarrow * \mathrm{NO} \rightarrow * \mathrm{~N} \rightarrow * \mathrm{NH} \rightarrow * \mathrm{NH}_{2} \rightarrow * \mathrm{NH}_{3} \rightarrow \mathrm{NH}_{3}(\mathrm{~g})$} \\
\hline Acidic solution & & Alkaline solution $^{a}$ & \\
\hline $\mathrm{NO}_{3}^{-}(1)+* \rightarrow * \mathrm{NO}_{3}+\mathrm{e}^{-}$ & $(r 1)$ & $\mathrm{NO}_{3}^{-}(1)+* \rightarrow * \mathrm{NO}_{3}+\mathrm{e}^{-}$ & $(r 1)$ \\
\hline$* \mathrm{NO}_{3}+2 \mathrm{H}^{+}+2 \mathrm{e}^{-} \rightarrow * \mathrm{NO}_{2}+\mathrm{H}_{2} \mathrm{O}$ & $(r 2)$ & $* \mathrm{NO}_{3}+\mathrm{H}_{2} \mathrm{O}+2 \mathrm{e}^{-} \rightarrow * \mathrm{NO}_{2}+2 \mathrm{OH}^{-}$ & $(r 2 a)$ \\
\hline$* \mathrm{NO}_{2}+2 \mathrm{H}^{+}+2 \mathrm{e}^{-} \rightarrow * \mathrm{NO}+\mathrm{H}_{2} \mathrm{O}$ & $(r 3)$ & $* \mathrm{NO}_{2}+\mathrm{H}_{2} \mathrm{O}+2 \mathrm{e}^{-} \rightarrow * \mathrm{NO}+2 \mathrm{OH}^{-}$ & (r3a) \\
\hline$* \mathrm{NO}+2 \mathrm{H}^{+}+2 \mathrm{e}^{-} \rightarrow * \mathrm{~N}+\mathrm{H}_{2} \mathrm{O}$ & $(r 4)$ & $* \mathrm{NO}+\mathrm{H}_{2} \mathrm{O}+2 \mathrm{e}^{-} \rightarrow * \mathrm{~N}+2 \mathrm{OH}^{-}$ & $(r 4 a)$ \\
\hline$* \mathrm{~N}+\mathrm{H}^{+}+\mathrm{e}^{-} \rightarrow * \mathrm{NH}$ & $(r 5)$ & $* \mathrm{~N}+\mathrm{H}_{2} \mathrm{O}+\mathrm{e}^{-} \rightarrow * \mathrm{NH}+\mathrm{OH}^{-}$ & $(r 5 \mathrm{a})$ \\
\hline$* \mathrm{NH}+\mathrm{H}^{+}+\mathrm{e}^{-} \rightarrow * \mathrm{NH}_{2}$ & $(r 6)$ & $* \mathrm{NH}+\mathrm{H}_{2} \mathrm{O}+\mathrm{e}^{-} \rightarrow * \mathrm{NH}_{2}+\mathrm{OH}^{-}$ & $(r 6 a)$ \\
\hline$* \mathrm{NH}_{2}+\mathrm{H}^{+}+\mathrm{e}^{-} \rightarrow * \mathrm{NH}_{3}$ & $(r 7)$ & $* \mathrm{NH}_{2}+\mathrm{H}_{2} \mathrm{O}+\mathrm{e}^{-} \rightarrow * \mathrm{NH}_{3}+\mathrm{OH}^{-}$ & $(r 7 \mathrm{a})$ \\
\hline${ }^{*} \mathrm{NH}_{3} \rightarrow *+\mathrm{NH}_{3}(\mathrm{~g})$ & $(r 8)$ & ${ }^{*} \mathrm{NH}_{3} \rightarrow{ }^{*}+\mathrm{NH}_{3}(\mathrm{~g})$ & $(r 8)$ \\
\hline $\mathrm{NO}_{3}^{-}+9 \mathrm{H}^{+}+8 \mathrm{e}^{-} \rightarrow \mathrm{NH}_{3}+6 \mathrm{H}_{2} \mathrm{O}$ & (total) & $\mathrm{NO}_{3}{ }^{-}+6 \mathrm{H}_{2} \mathrm{O}+8 \mathrm{e}^{-} \rightarrow \mathrm{NH}_{3}+9 \mathrm{OH}^{-}$ & (total) \\
\hline
\end{tabular}

${ }^{a}$ In thermodynamic calculation, the total energy of negatively charged radical $\mathrm{OH}^{-}$is difficult to treat, and is approached using the stable molecules $\mathrm{H}_{2} \mathrm{O}$ and $\mathrm{H}_{2}$ instead. $E\left(\mathrm{OH}^{-}\right)=E\left(\mathrm{H}_{2} \mathrm{O}\right)-1 / 2 E\left(\mathrm{H}_{2}\right)$, $\mathrm{TS}\left(\mathrm{OH}^{-}\right)=\mathrm{TS}\left(\mathrm{H}_{2} \mathrm{O}\right)-1 / 2 \mathrm{TS}\left(\mathrm{H}_{2}\right)$. By this approach, the thermodynamics of intermediates in alkaline solution are equal to those in acidic solution.

Taking equation ( $r 2 \mathrm{a})$ as an example, the Gibbs free energy is calculated by

$\Delta \mathrm{G}_{\mathrm{r} 2 \mathrm{a}}=\Delta \mathrm{E}-\mathrm{T} \Delta \mathrm{S}=E\left(* \mathrm{NO}_{2}\right)+2 E\left(\mathrm{OH}^{-}\right)-E\left(* \mathrm{NO}_{3}\right)-E\left(\mathrm{H}_{2} \mathrm{O}\right)-\left[2 \mathrm{TS}\left(\mathrm{OH}^{-}\right)-\mathrm{TS}\left(\mathrm{H}_{2} \mathrm{O}\right)\right]$

$=E\left(* \mathrm{NO}_{2}\right)+2\left[E\left(\mathrm{H}_{2} \mathrm{O}\right)-1 / 2 E\left(\mathrm{H}_{2}\right)\right]-E\left(* \mathrm{NO}_{3}\right)-E\left(\mathrm{H}_{2} \mathrm{O}\right)-\left[2\left(\mathrm{TS}\left(\mathrm{H}_{2} \mathrm{O}\right)-1 / 2 \mathrm{TS}\left(\mathrm{H}_{2}\right)\right)-\mathrm{TS}\left(\mathrm{H}_{2} \mathrm{O}\right)\right]$

$=E\left(* \mathrm{NO}_{2}\right)+E\left(\mathrm{H}_{2} \mathrm{O}\right)-E\left(\mathrm{H}_{2}\right)-E\left(* \mathrm{NO}_{3}\right)-\left[\mathrm{TS}\left(\mathrm{H}_{2} \mathrm{O}\right)-1 / 2 \mathrm{TS}\left(\mathrm{H}_{2}\right)\right]$

$=\Delta \mathrm{G}_{\mathrm{r} 2}$ 
Table S5. NRA2 reaction steps in acidic solution and alkaline solution

\begin{tabular}{|c|c|c|c|}
\hline \multicolumn{4}{|c|}{ NRA2: $\mathrm{NO}_{3}{ }^{-} \rightarrow * \mathrm{NO}_{3}{ }^{-} \rightarrow * \mathrm{NO}_{3} \rightarrow * \mathrm{NO}_{2} \rightarrow * \mathrm{NO} \rightarrow * \mathrm{NOH} \rightarrow * \mathrm{NHOH} \rightarrow * \mathrm{NH}_{2} \mathrm{OH} \rightarrow * \mathrm{NH}_{2} \rightarrow * \mathrm{NH}_{3} \rightarrow \mathrm{NH}_{3}(\mathrm{~g})$} \\
\hline Acidic solution & & Alkaline solution & \\
\hline $\mathrm{NO}_{3}^{-}(1)+* \rightarrow * \mathrm{NO}_{3}+\mathrm{e}^{-}$ & $(r 1)$ & $\mathrm{NO}_{3}^{-}(1)+* \rightarrow * \mathrm{NO}_{3}+\mathrm{e}^{-}$ & $(r 1)$ \\
\hline$* \mathrm{NO}_{3}+2 \mathrm{H}^{+}+2 \mathrm{e}^{-} \rightarrow * \mathrm{NO}_{2}+\mathrm{H}_{2} \mathrm{O}$ & $(r 2)$ & $* \mathrm{NO}_{3}+\mathrm{H}_{2} \mathrm{O}+2 \mathrm{e}^{-} \rightarrow * \mathrm{NO}_{2}+2 * \mathrm{OH}^{-}$ & $(r 2 \mathrm{a})$ \\
\hline$* \mathrm{NO}_{2}+2 \mathrm{H}^{+}+2 \mathrm{e}^{-} \rightarrow * \mathrm{NO}+\mathrm{H}_{2} \mathrm{O}$ & $(r 3)$ & $* \mathrm{NO}_{2}+\mathrm{H}_{2} \mathrm{O}+2 \mathrm{e}^{-} \rightarrow * \mathrm{NO}+2 \mathrm{OH}^{-}$ & $(r 3 a)$ \\
\hline$* \mathrm{NO}+\mathrm{H}^{+}+\mathrm{e}^{-} \rightarrow * \mathrm{NOH}$ & $(r 9)$ & $* \mathrm{NO}+\mathrm{H}_{2} \mathrm{O}+\mathrm{e}^{-} \rightarrow * \mathrm{NOH}+\mathrm{OH}^{-}$ & $(r 9 a)$ \\
\hline$* \mathrm{NOH}+\mathrm{H}^{+}+\mathrm{e}^{-} \rightarrow * \mathrm{NHOH}$ & $(r 10)$ & $* \mathrm{NOH}+\mathrm{H}_{2} \mathrm{O}+\mathrm{e}^{-} \rightarrow * \mathrm{NHOH}+\mathrm{OH}^{-}$ & $(r 10 \mathrm{a})$ \\
\hline$* \mathrm{NHOH}+\mathrm{H}^{+}+\mathrm{e}^{-} \rightarrow * \mathrm{NH}_{2} \mathrm{OH}$ & $(r 11)$ & $* \mathrm{NHOH}+\mathrm{H}_{2} \mathrm{O}+\mathrm{e}^{-} \rightarrow * \mathrm{NH}_{2} \mathrm{OH}+\mathrm{OH}^{-}$ & $(r 11 \mathrm{a})$ \\
\hline$* \mathrm{NH}_{2} \mathrm{OH}+\mathrm{H}^{+}+\mathrm{e}^{-} \rightarrow * \mathrm{NH}_{2}+\mathrm{H}_{2} \mathrm{O}$ & $(r 12)$ & $* \mathrm{NH}_{2} \mathrm{OH}+\mathrm{e}^{-} \rightarrow * \mathrm{NH}_{2}+\mathrm{OH}^{-}$ & $(r 12 \mathrm{a})$ \\
\hline$* \mathrm{NH}_{2}+\mathrm{H}^{+}+\mathrm{e}^{-} \rightarrow * \mathrm{NH}_{3}$ & $(r 7)$ & $* \mathrm{NH}_{2}+\mathrm{H}_{2} \mathrm{O}+\mathrm{e}^{-} \rightarrow * \mathrm{NH}_{3}+\mathrm{OH}^{-}$ & $(r 7 \mathrm{a})$ \\
\hline$* \mathrm{NH}_{3} \rightarrow *+\mathrm{NH}_{3}(\mathrm{~g})$ & $(r 8)$ & $* \mathrm{NH}_{3} \rightarrow *+\mathrm{NH}_{3}(\mathrm{~g})$ & $(r 8)$ \\
\hline $\mathrm{NO}_{3}^{-}+9 \mathrm{H}^{+}+8 \mathrm{e}^{-} \rightarrow \mathrm{NH}_{3}+6 \mathrm{H}_{2} \mathrm{O}$ & (total) & $\mathrm{NO}_{3}^{-}+6 \mathrm{H}_{2} \mathrm{O}+8 \mathrm{e}^{-} \rightarrow \mathrm{NH}_{3}+9 \mathrm{OH}^{-}$ & (total) \\
\hline
\end{tabular}

Table S6. NRA3 reaction steps in acidic solution and alkaline solution

\begin{tabular}{|c|c|c|c|}
\hline \multicolumn{4}{|c|}{$\mathrm{NRA} 3: * \rightarrow * \mathrm{NO}_{3} \rightarrow * \mathrm{NO}_{2} \rightarrow * \mathrm{NO} \rightarrow * \mathrm{NOH} \rightarrow * \mathrm{NHOH} \rightarrow * \mathrm{NH} \rightarrow * \mathrm{NH}_{2} \rightarrow * \mathrm{NH}_{3} \rightarrow \mathrm{NH}_{3}(\mathrm{~g})$} \\
\hline Acidic solution & & Alkaline solution & \\
\hline $\mathrm{NO}_{3}^{-}(1)+* \rightarrow * \mathrm{NO}_{3}+\mathrm{e}^{-}$ & $(r 1)$ & $\mathrm{NO}_{3}^{-}(1)+* \rightarrow * \mathrm{NO}_{3}+\mathrm{e}^{-}$ & $(r 1)$ \\
\hline$* \mathrm{NO}_{3}+2 \mathrm{H}^{+}+2 \mathrm{e}^{-} \rightarrow * \mathrm{NO}_{2}+\mathrm{H}_{2} \mathrm{O}$ & $(r 2)$ & $* \mathrm{NO}_{3}+\mathrm{H}_{2} \mathrm{O}+2 \mathrm{e}^{-} \rightarrow * \mathrm{NO}_{2}+2 * \mathrm{OH}^{-}$ & $(r 2 a)$ \\
\hline$* \mathrm{NO}_{2}+2 \mathrm{H}^{+}+2 \mathrm{e}^{-} \rightarrow * \mathrm{NO}+\mathrm{H}_{2} \mathrm{O}$ & $(r 3)$ & $* \mathrm{NO}_{2}+\mathrm{H}_{2} \mathrm{O}+2 \mathrm{e}^{-} \rightarrow * \mathrm{NO}+2 \mathrm{OH}^{-}$ & $(r 3 a)$ \\
\hline$* \mathrm{NO}+\mathrm{H}^{+}+\mathrm{e}^{-} \rightarrow * \mathrm{NOH}$ & $(r 9)$ & $* \mathrm{NO}+\mathrm{H}_{2} \mathrm{O}+\mathrm{e}^{-} \rightarrow * \mathrm{NOH}+\mathrm{OH}^{-}$ & $(r 9 \mathrm{a})$ \\
\hline$* \mathrm{NOH}+\mathrm{H}^{+}+\mathrm{e}^{-} \rightarrow * \mathrm{NHOH}$ & $(r 10)$ & $* \mathrm{NOH}+\mathrm{H}_{2} \mathrm{O}+\mathrm{e}^{-} \rightarrow * \mathrm{NHOH}+\mathrm{OH}^{-}$ & $(r 10 \mathrm{a})$ \\
\hline$* \mathrm{NHOH}+\mathrm{H}^{+}+\mathrm{e}^{-} \rightarrow * \mathrm{NH}+\mathrm{H}_{2} \mathrm{O}$ & $(r 13)$ & $* \mathrm{NHOH}+\mathrm{e}^{-} \rightarrow * \mathrm{NH}+\mathrm{OH}^{-}$ & $(r 13 a)$ \\
\hline$* \mathrm{NH}+\mathrm{H}^{+}+\mathrm{e}^{-} \rightarrow * \mathrm{NH}_{2}$ & $(r 6)$ & $* \mathrm{NH}+\mathrm{H}_{2} \mathrm{O}+\mathrm{e}^{-} \rightarrow * \mathrm{NH}_{2}+\mathrm{OH}^{-}$ & $(r 6 \mathrm{a})$ \\
\hline$* \mathrm{NH}_{2}+\mathrm{H}^{+}+\mathrm{e}^{-} \rightarrow * \mathrm{NH}_{3}$ & $(r 7)$ & $* \mathrm{NH}_{2}+\mathrm{H}_{2} \mathrm{O}+\mathrm{e}^{-} \rightarrow * \mathrm{NH}_{3}+\mathrm{OH}^{-}$ & $(r 7 a)$ \\
\hline$* \mathrm{NH}_{3} \rightarrow *+\mathrm{NH}_{3}(\mathrm{~g})$ & $(r 8)$ & $* \mathrm{NH}_{3} \rightarrow *+\mathrm{NH}_{3}(\mathrm{~g})$ & $(r 8)$ \\
\hline $\mathrm{NO}_{3}{ }^{-}+9 \mathrm{H}^{+}+8 \mathrm{e}^{-} \rightarrow \mathrm{NH}_{3}+6 \mathrm{H}_{2} \mathrm{O}$ & (total) & $\mathrm{NO}_{3}^{-}+6 \mathrm{H}_{2} \mathrm{O}+8 \mathrm{e}^{-} \rightarrow \mathrm{NH}_{3}+9 \mathrm{OH}^{-}$ & (total) \\
\hline
\end{tabular}




\section{Part II Supplementary Tables and Figures}

Table S7. Enthalpic contribution of gas corrections for $\mathrm{H}_{2} \mathrm{O}, \mathrm{H}_{2}$ and $\mathrm{NH}_{3}$ used in the Gibbs free energy calculations

\begin{tabular}{|c|c|}
\hline Molecule & $\mathrm{T} \Delta \mathrm{S}$ \\
\hline $\mathrm{H}_{2} \mathrm{O}$ & $0.67 \mathrm{eV}^{\mathrm{a}}$ \\
\hline $\mathrm{H}_{2}$ & $0.41 \mathrm{eV}^{\mathrm{a}}$ \\
\hline $\mathrm{NH}_{3}$ & $0.60 \mathrm{eV}^{\mathrm{b}}$ \\
\hline
\end{tabular}

${ }^{a}$ Data from Norskov's work ${ }^{1}$

${ }^{\mathrm{b}}$ Data from Lange's handbook, ${ }^{2}$ Table 1.56, Page 1.238, T $\Delta \mathrm{S}\left(\mathrm{NH}_{3}\right)=192.78 \mathrm{Jdeg}^{-1} \mathrm{~mol}^{-1} * 298.15$ $\mathrm{K}=0.60 \mathrm{eV}$

Table S8. Calculated Gibbs free energies (in eV) of NRA1 on $\mathrm{Cu}(111)$ at different $\mathrm{pH}$

\begin{tabular}{|c|c|c|c|}
\hline & $\mathrm{pH}=0$ & $\mathrm{pH}=7$ & $\mathrm{pH}=14$ \\
\hline$\Delta \mathrm{G}_{1}$ & 0.06 & 0.06 & 0.06 \\
\hline$\Delta \mathrm{G}_{2}$ & -1.81 & -0.98 & -0.16 \\
\hline$\Delta \mathrm{G}_{3}$ & -1.16 & -0.34 & 0.49 \\
\hline$\Delta \mathrm{G}_{4}$ & -0.85 & -0.02 & 0.81 \\
\hline$\Delta \mathrm{G}_{5}$ & -1.20 & -0.79 & -0.38 \\
\hline$\Delta \mathrm{G}_{6}$ & -0.42 & -0.01 & 0.40 \\
\hline$\Delta \mathrm{G}_{7}$ & -0.53 & -0.12 & 0.30 \\
\hline$\Delta \mathrm{G}_{8}$ & 0.37 & 0.37 & 0.37 \\
\hline
\end{tabular}

Table S9. Calculated Gibbs free energies (in $\mathrm{eV}$ ) of NRA2 on $\mathrm{Cu}(111)$ at different $\mathrm{pH}$

\begin{tabular}{|c|c|c|c|}
\hline & $\mathrm{pH}=0$ & $\mathrm{pH}=7$ & $\mathrm{pH}=14$ \\
\hline$\Delta \mathrm{G}_{1}$ & 0.06 & 0.06 & 0.06 \\
\hline$\Delta \mathrm{G}_{2}$ & -1.81 & -0.98 & -0.16 \\
\hline$\Delta \mathrm{G}_{3}$ & -1.16 & -0.33 & 0.49 \\
\hline$\Delta \mathrm{G}_{4}$ & 0.08 & 0.50 & 0.91 \\
\hline$\Delta \mathrm{G}_{5}$ & -0.27 & 0.14 & 0.55 \\
\hline$\Delta \mathrm{G}_{6}$ & -0.18 & 0.23 & 0.64 \\
\hline
\end{tabular}




\begin{tabular}{|c|c|c|c|}
\hline$\Delta \mathrm{G}_{7}$ & -2.10 & -1.69 & -1.28 \\
\hline$\Delta \mathrm{G}_{8}$ & -0.53 & -0.12 & 0.30 \\
\hline$\Delta \mathrm{G}_{9}$ & 0.37 & 0.37 & 0.37 \\
\hline
\end{tabular}

Table S10. Calculated Gibbs free energies of NRA3 on $\mathrm{Cu}(111)$ at different $\mathrm{pH}$

\begin{tabular}{|c|c|c|c|}
\hline & $\mathrm{pH}=0$ & $\mathrm{pH}=7$ & $\mathrm{pH}=14$ \\
\hline$\Delta \mathrm{G}_{1}$ & 0.06 & 0.06 & 0.06 \\
\hline$\Delta \mathrm{G}_{2}$ & -1.81 & -0.98 & -0.16 \\
\hline$\Delta \mathrm{G}_{3}$ & -1.16 & -0.34 & 0.49 \\
\hline$\Delta \mathrm{G}_{4}$ & 0.08 & 0.50 & 0.91 \\
\hline$\Delta \mathrm{G}_{5}$ & -0.27 & 0.14 & 0.55 \\
\hline$\Delta \mathrm{G}_{6}$ & -1.86 & -1.45 & -1.04 \\
\hline$\Delta \mathrm{G}_{7}$ & -0.42 & -0.01 & 0.40 \\
\hline$\Delta \mathrm{G}_{8}$ & -0.53 & -0.12 & 0.30 \\
\hline$\Delta \mathrm{G}_{9}$ & 0.37 & 0.37 & 0.37 \\
\hline
\end{tabular}

Table S11. Calculated Gibbs free energies of NRA3 on $\mathrm{Cu}(100)$ at different $\mathrm{pH}$

\begin{tabular}{|c|c|c|c|}
\hline & $\mathrm{pH}=0$ & $\mathrm{pH}=7$ & $\mathrm{pH}=14$ \\
\hline$\Delta \mathrm{G}_{1}$ & -0.34 & -0.34 & -0.34 \\
\hline$\Delta \mathrm{G}_{2}$ & -1.74 & -0.91 & -0.08 \\
\hline$\Delta \mathrm{G}_{3}$ & -0.76 & 0.07 & 0.89 \\
\hline$\Delta \mathrm{G}_{4}$ & 0.31 & 0.72 & 1.14 \\
\hline$\Delta \mathrm{G}_{5}$ & -0.83 & -0.42 & -0.00 \\
\hline$\Delta \mathrm{G}_{6}$ & -2.08 & -1.67 & -1.26 \\
\hline$\Delta \mathrm{G}_{7}$ & -0.24 & 0.17 & 0.59 \\
\hline$\Delta \mathrm{G}_{8}$ & -0.12 & 0.29 & 0.71 \\
\hline$\Delta \mathrm{G}_{9}$ & 0.25 & 0.25 & 0.25 \\
\hline
\end{tabular}


Table S12. Calculated Gibbs free energies of NRA3 on $\mathrm{Cu}(110)$ at different $\mathrm{pH}$

\begin{tabular}{|c|c|c|c|}
\hline & $\mathrm{pH}=0$ & $\mathrm{pH}=7$ & $\mathrm{pH}=14$ \\
\hline$\Delta \mathrm{G}_{1}$ & -0.44 & -0.44 & -0.44 \\
\hline$\Delta \mathrm{G}_{2}$ & -1.79 & -0.96 & -0.13 \\
\hline$\Delta \mathrm{G}_{3}$ & -0.66 & 0.17 & 1.00 \\
\hline$\Delta \mathrm{G}_{4}$ & 0.38 & 0.80 & 1.21 \\
\hline$\Delta \mathrm{G}_{5}$ & -0.84 & -0.43 & -0.01 \\
\hline$\Delta \mathrm{G}_{6}$ & -1.49 & -1.08 & -0.66 \\
\hline$\Delta \mathrm{G}_{7}$ & -0.96 & -0.54 & -0.13 \\
\hline$\Delta \mathrm{G}_{8}$ & -0.30 & 0.12 & 0.53 \\
\hline$\Delta \mathrm{G}_{9}$ & 0.54 & 0.54 & 0.54 \\
\hline
\end{tabular}

Table S13. The correction of zero-point energy and entropy of adsorption species and molecules involved in NRA. $T$ is the room temperature $(298.15 \mathrm{~K})$.

\begin{tabular}{|c|c|}
\hline Species & ZPE $(\mathrm{eV})$ \\
\hline$* \mathrm{NO}_{3}$ & 0.42 \\
\hline$* \mathrm{NO}_{2}$ & 0.28 \\
\hline$* \mathrm{NO}$ & 0.17 \\
\hline$* \mathrm{~N}$ & 0.08 \\
\hline$* \mathrm{NH}$ & 0.38 \\
\hline$* \mathrm{NH}_{2}$ & 0.69 \\
\hline$* \mathrm{NH}_{3}$ & 1.00 \\
\hline$* \mathrm{NOH}^{2}$ & 0.45 \\
\hline$* \mathrm{NH}_{2} \mathrm{OH}$ & 1.12 \\
\hline$* \mathrm{NO}_{2} \mathrm{H}$ & 0.59 \\
\hline$* \mathrm{NHO}$ & 0.43 \\
\hline$* \mathrm{NHOH}^{*} \mathrm{H}$ & 0.79 \\
\hline $\mathrm{H}_{2}(\mathrm{~g})$ & 0.17 \\
\hline $\mathrm{H}_{2} \mathrm{O}(\mathrm{g})$ & 0.27 \\
\hline $\mathrm{NH}_{3}(\mathrm{~g})$ & 0.57 \\
\hline & 0.91 \\
\hline
\end{tabular}




\section{Activation energy of NRA on $\mathrm{Cu}(111)$ in $\mathrm{eV}$}

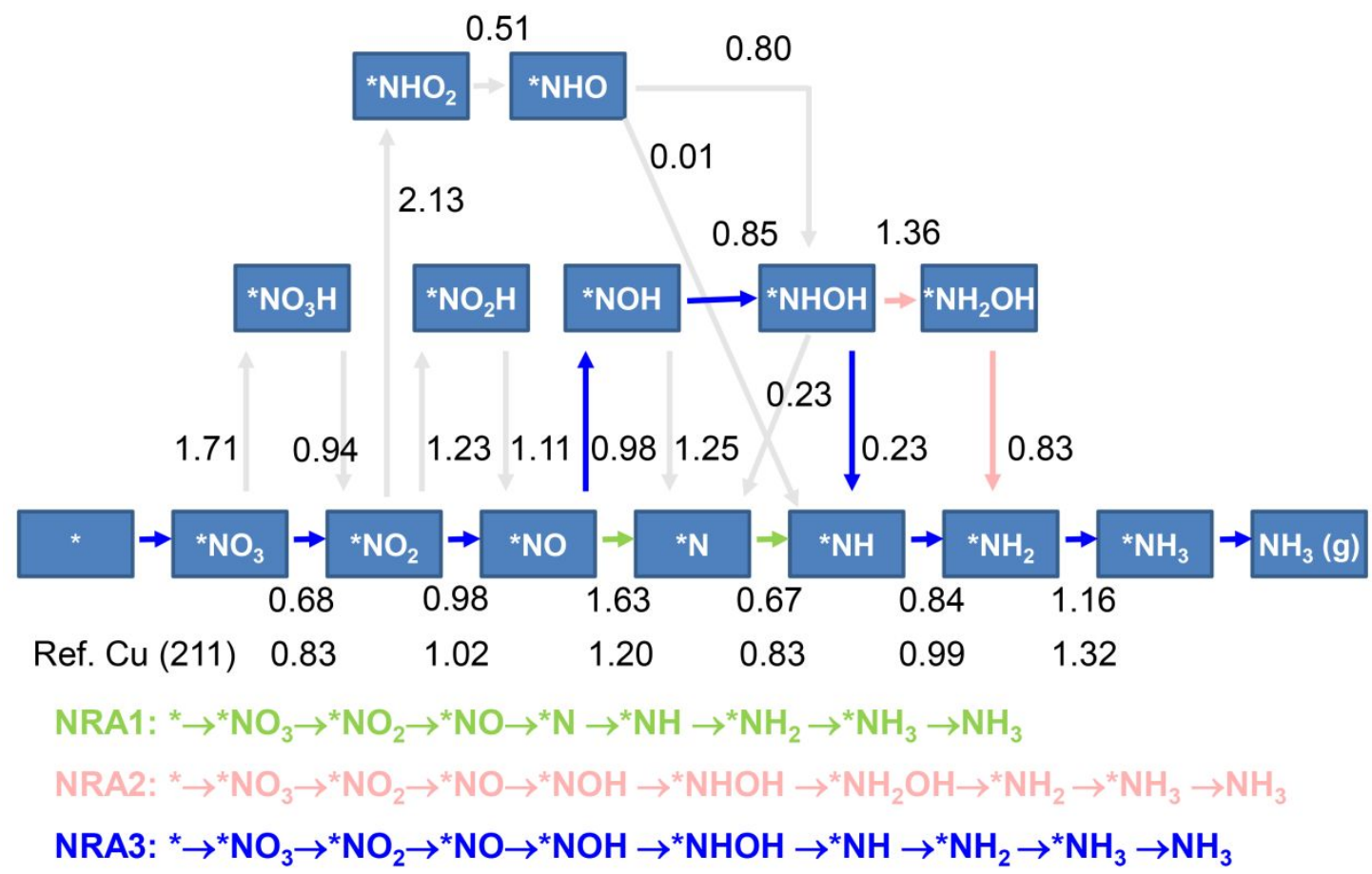

Figure S1. The overview of the reaction kinetics on $\mathrm{Cu}(111)$ for NRA. The Activation energy of each elementary step is given in $\mathrm{eV}$. The available activation energy data for NRA1 on $\mathrm{Cu}(211)^{3}$ is also compared with the data calculated on $\mathrm{Cu}(111) .{ }^{*} \mathrm{NO} \rightarrow{ }^{*} \mathrm{~N}$ is prohibited by the high activation energy in the room termperature electrochemical condition. Combined the thermodynacmis and kinetics data, NRA3 is the most favorable pathway for NRA.

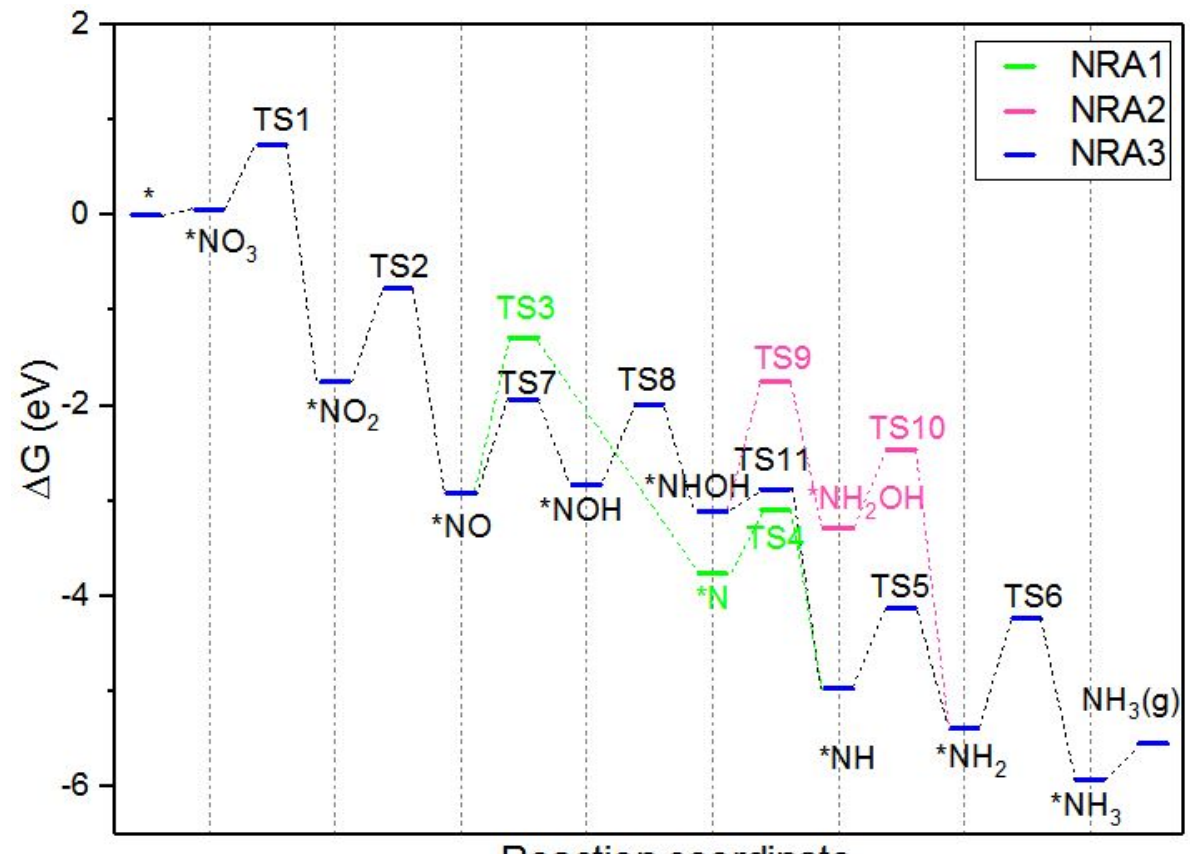

Reaction coordinate

Figure S2. Potential energy diagrams, and transition states of three pathways for the conversion of $\mathrm{NO}_{3}{ }^{-}$to $\mathrm{NH}_{3}$ on $\mathrm{Cu}(111)$ surface. 


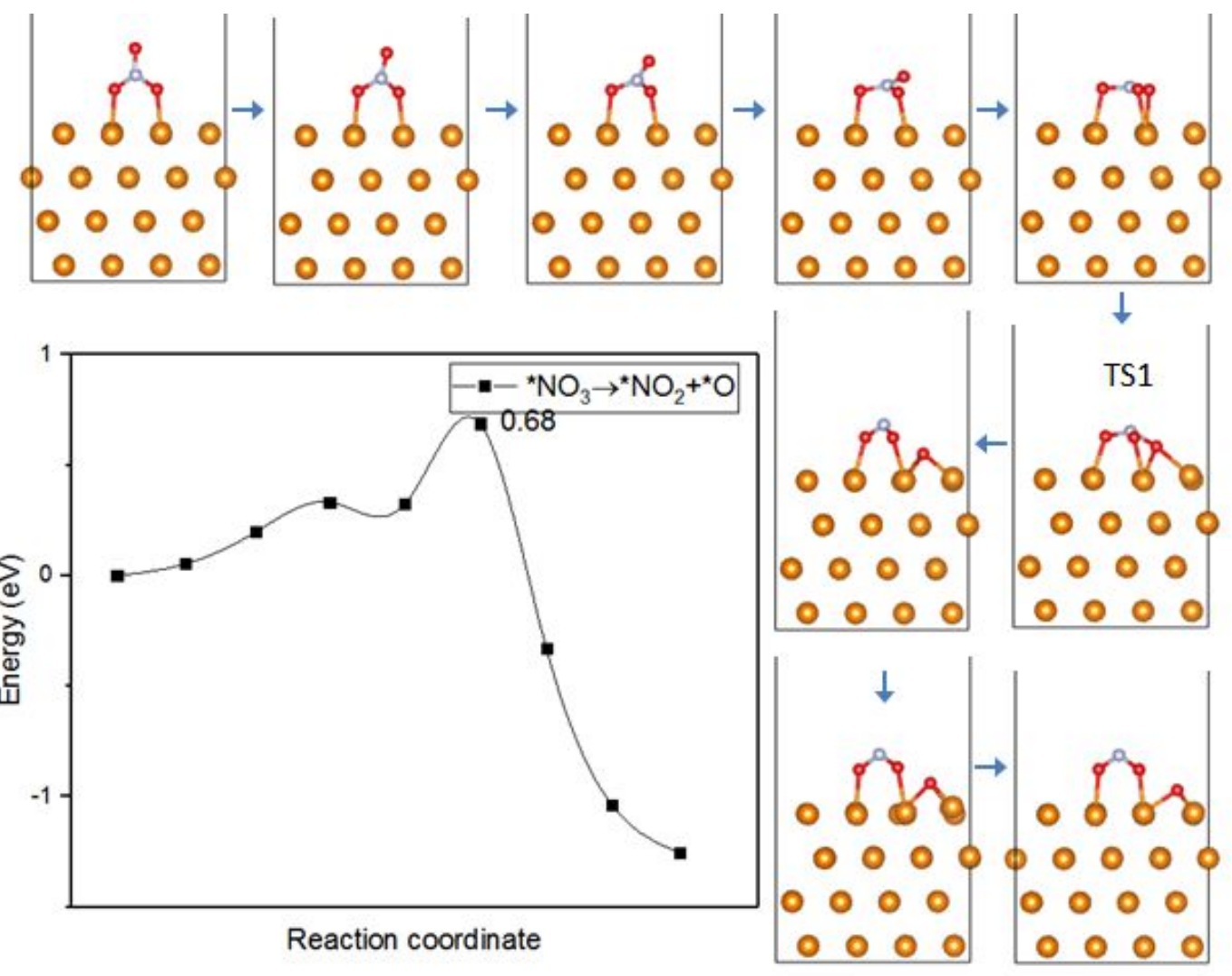

Figure S3. Minimum energy path and activation barrier of elementary step $* \mathrm{NO}_{3} \rightarrow \mathrm{NO}_{2}+* \mathrm{O}$.

TS1 stands for the transition state.

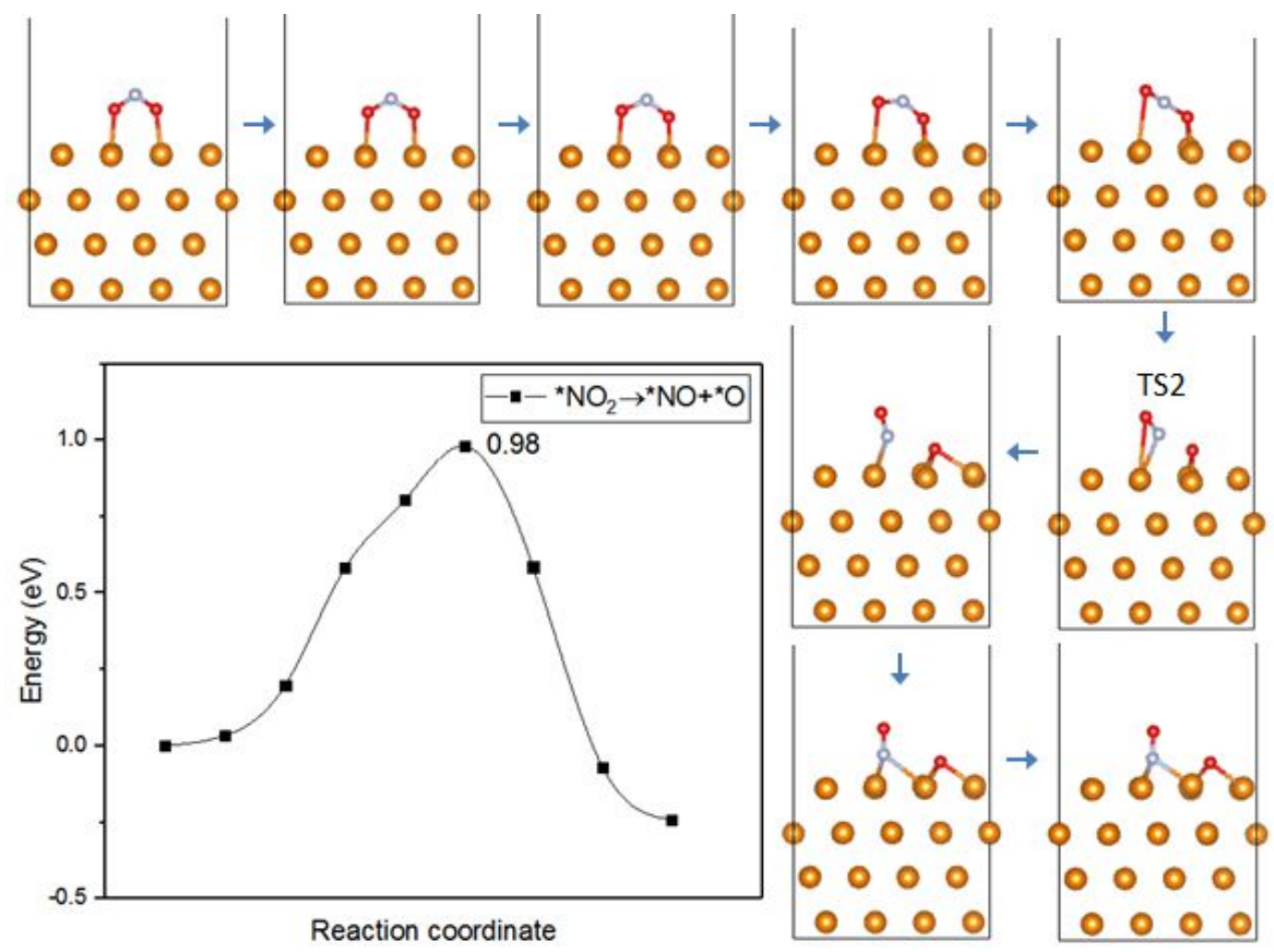

Figure S4. Minimum energy path and activation barrier of elementary step $* \mathrm{NO}_{2} \rightarrow * \mathrm{NO}+* \mathrm{O}$. TS2 stands for the transition state. 


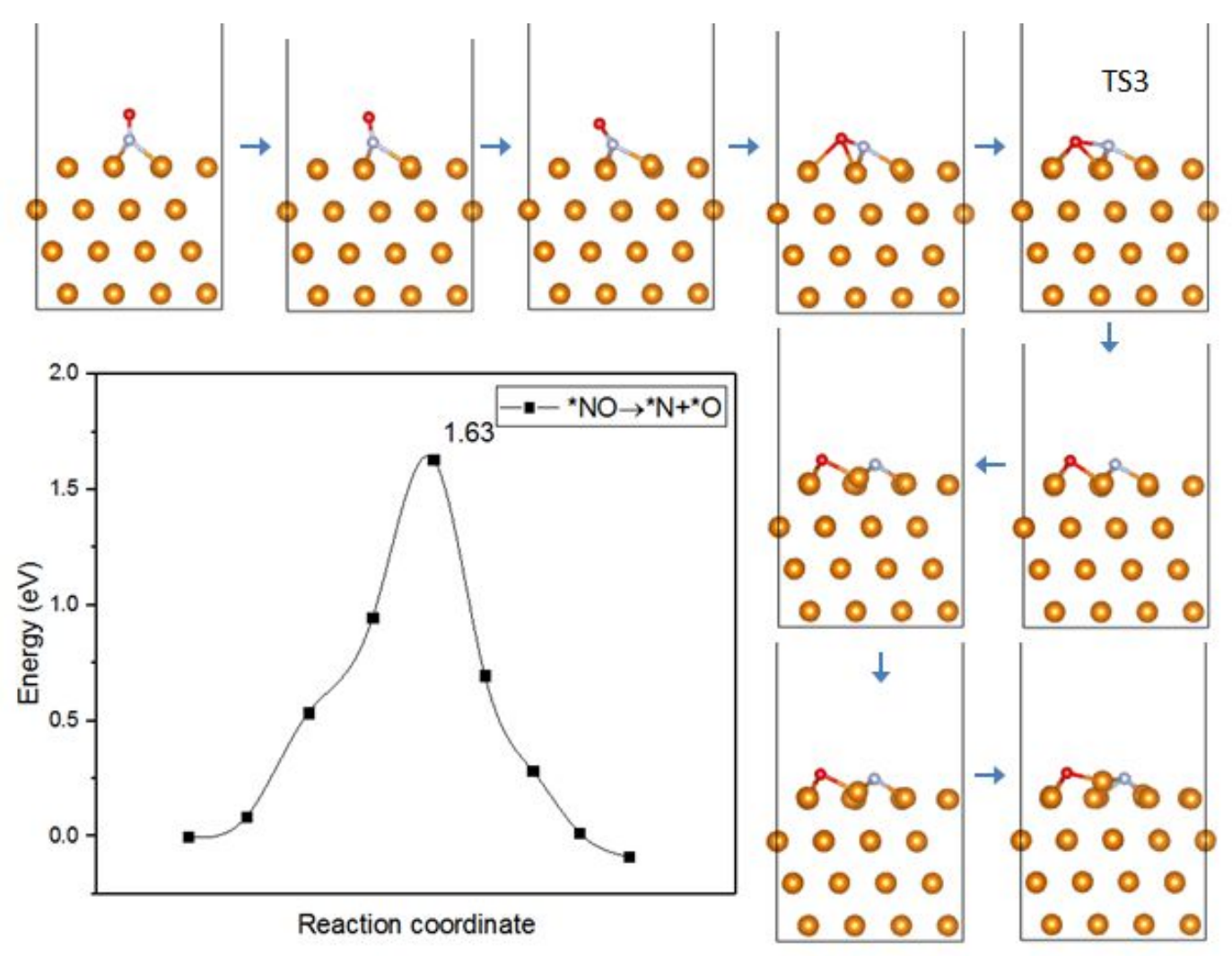

Figure S5. Minimum energy path and activation barrier of elementary step $* \mathrm{NO} \rightarrow{ }^{*} \mathrm{~N}+{ }^{*} \mathrm{O}$. TS3 stands for the transition state.

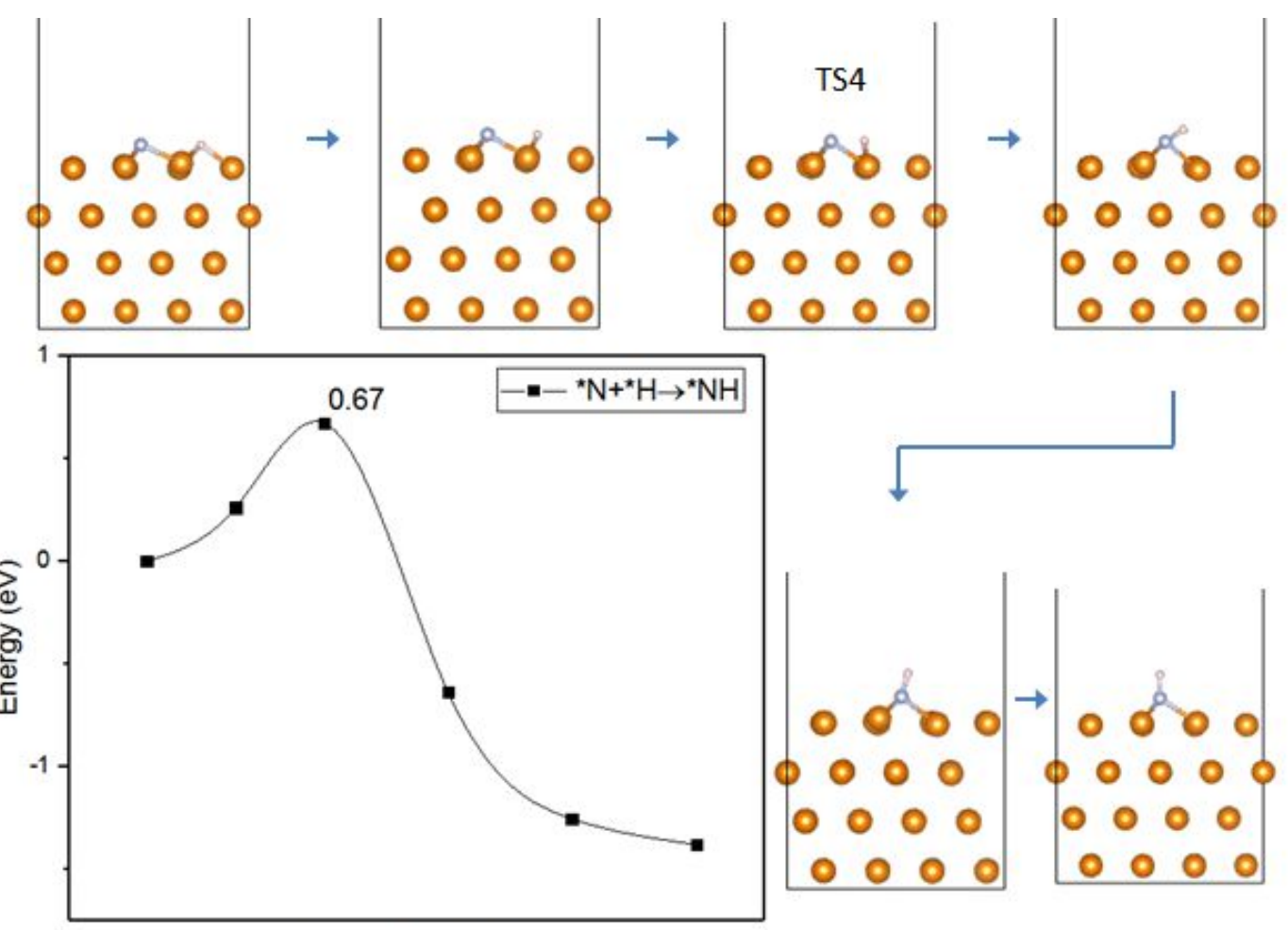

Reaction coordinate

Figure S6. Minimum energy path and activation barrier of elementary step $* \mathrm{~N}+* \mathrm{H} \rightarrow{ }^{*} \mathrm{NH}$. TS4 stands for the transition state. 


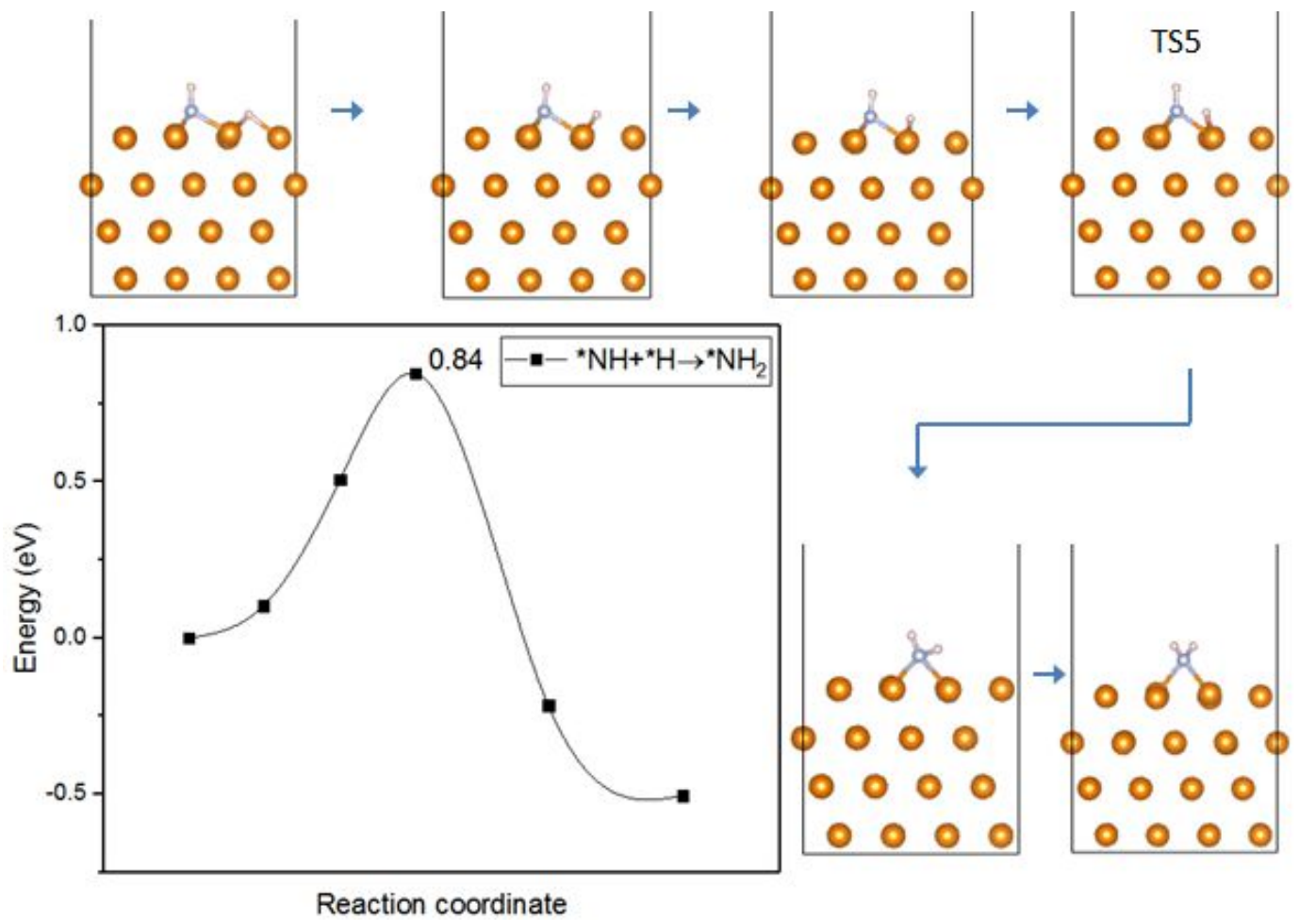

Figure S7. Minimum energy path and activation barrier of elementary step $* \mathrm{NH}+* \mathrm{H} \rightarrow * \mathrm{NH}_{2}$. TS5 stands for the transition state.

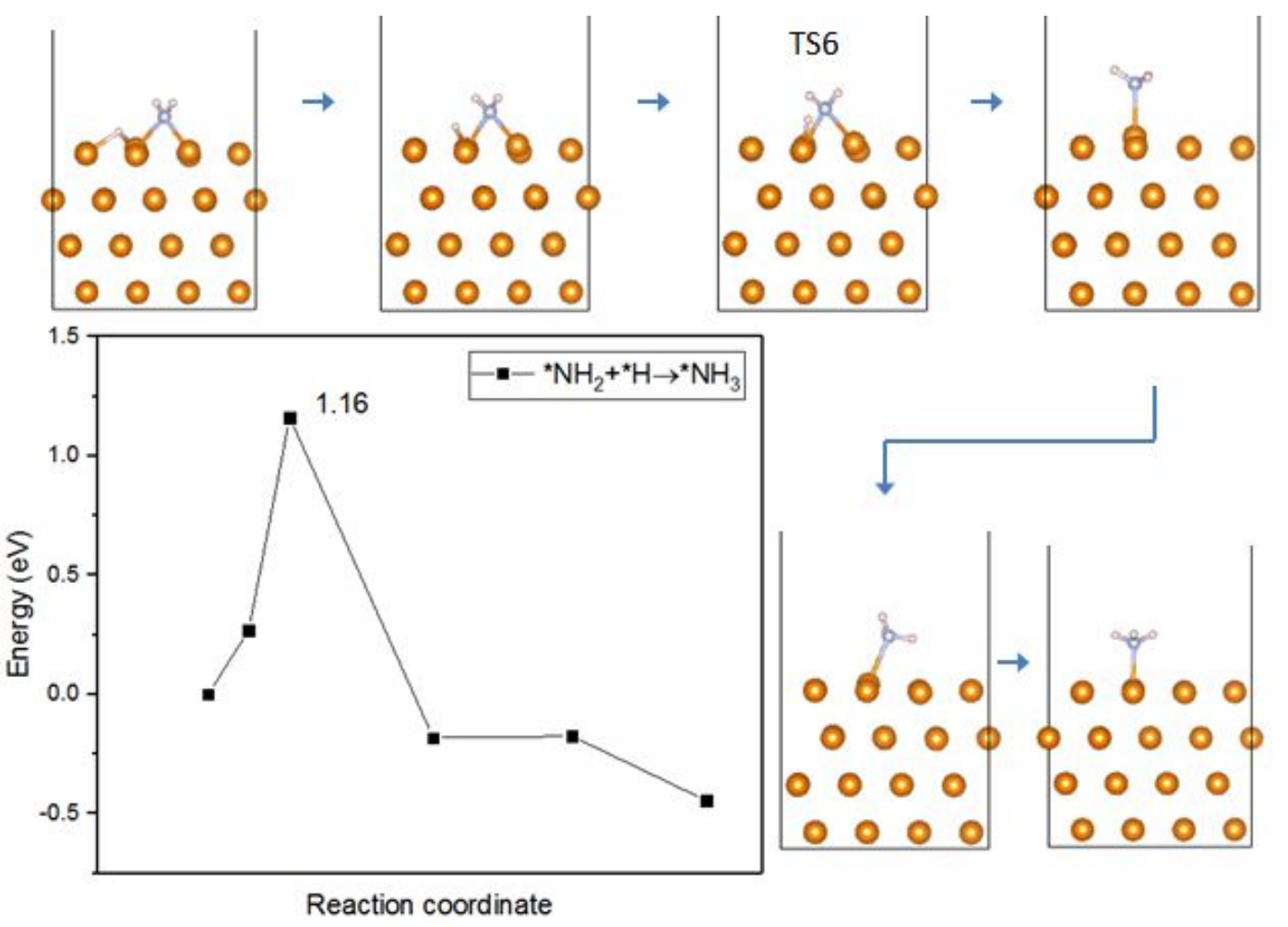

Figure S8. Minimum energy path and activation barrier of elementary step $* \mathrm{NH}_{2}+* \mathrm{H} \rightarrow{ }^{*} \mathrm{NH}_{3}$. TS6 stands for the transition state. 


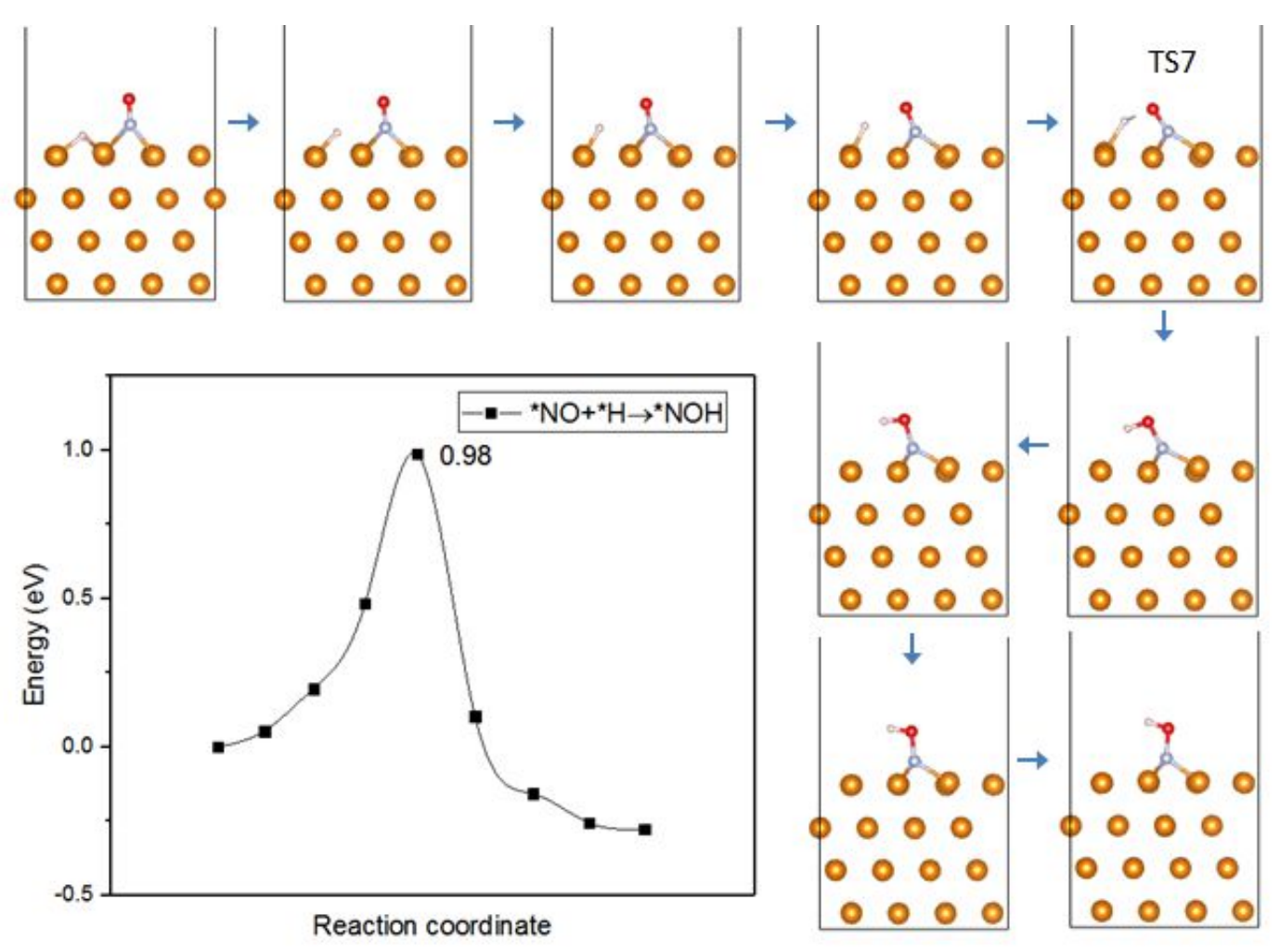

Figure S9. Minimum energy path and activation barrier of elementary step $* \mathrm{NO}+{ }^{*} \mathrm{H} \rightarrow * \mathrm{NOH}$.

TS7 stands for the transition state.

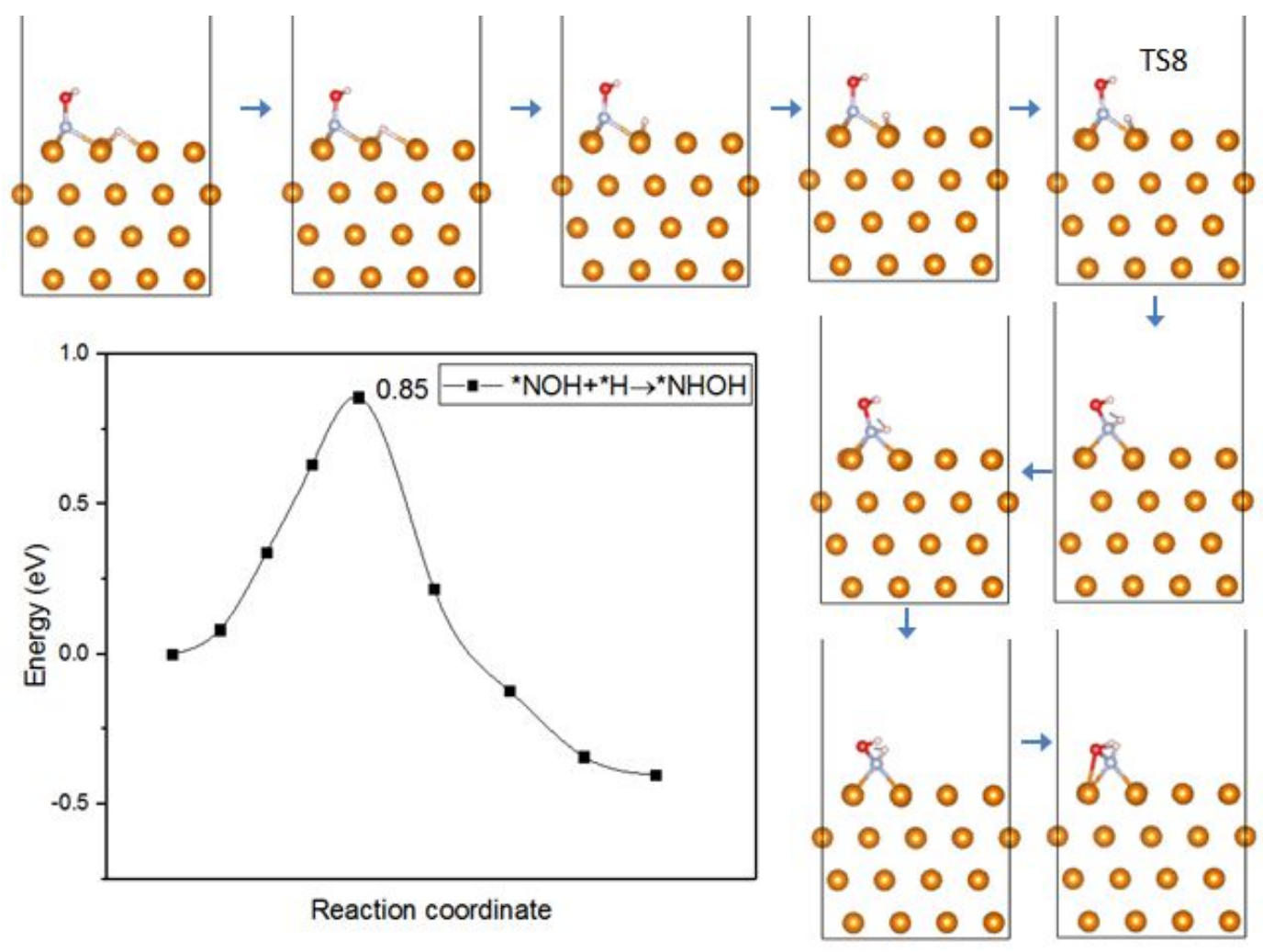

Figure S10. Minimum energy path and activation barrier of elementary step $* \mathrm{NOH}+* \mathrm{H} \rightarrow * \mathrm{NHOH}$. TS8 stands for the transition state. 


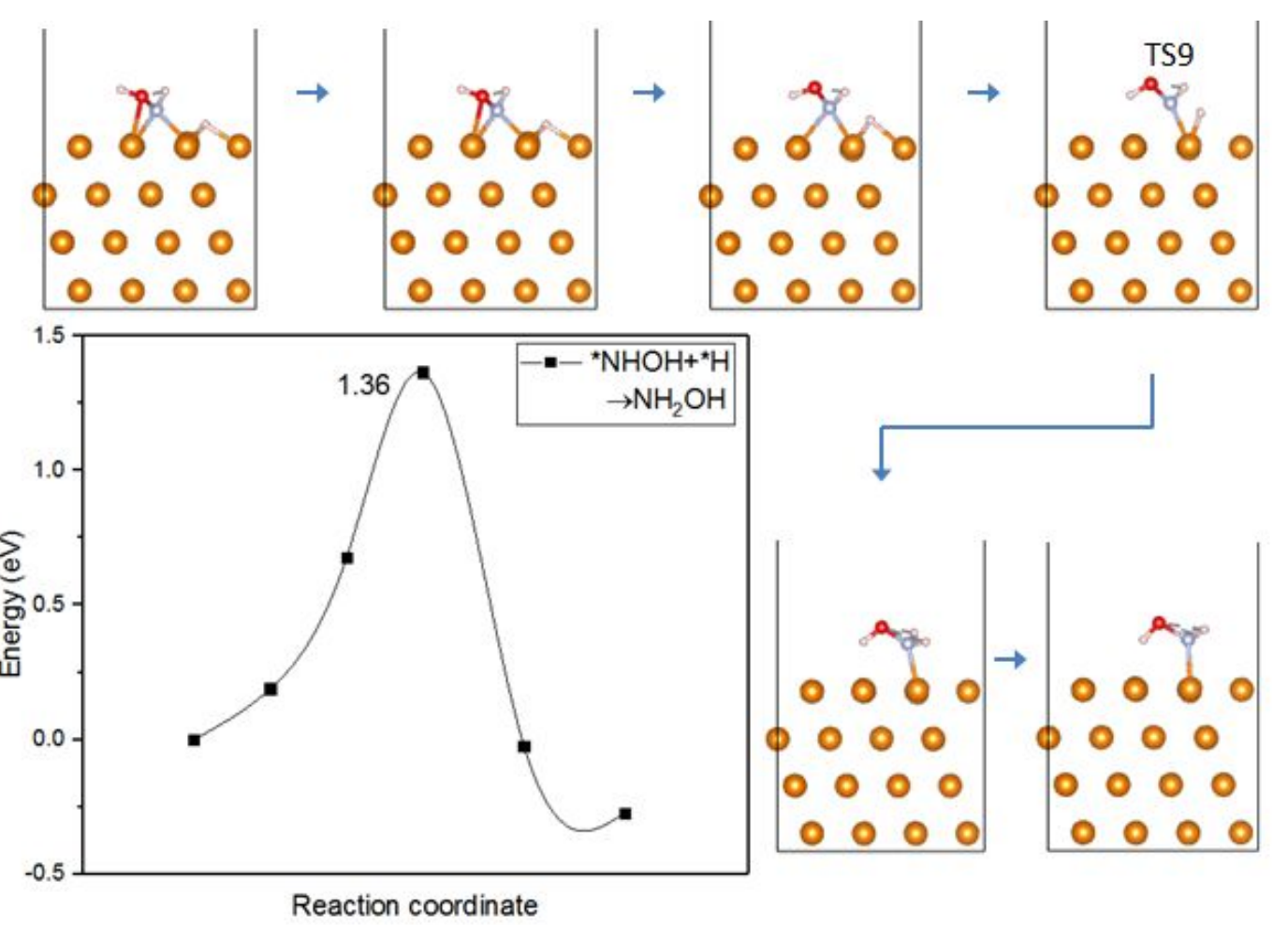

Figure S11. Minimum energy path and activation barrier of elementary step $* \mathrm{NHOH}+* \mathrm{H} \rightarrow{ }^{*} \mathrm{NH}_{2} \mathrm{OH}$. TS9 stands for the transition state.

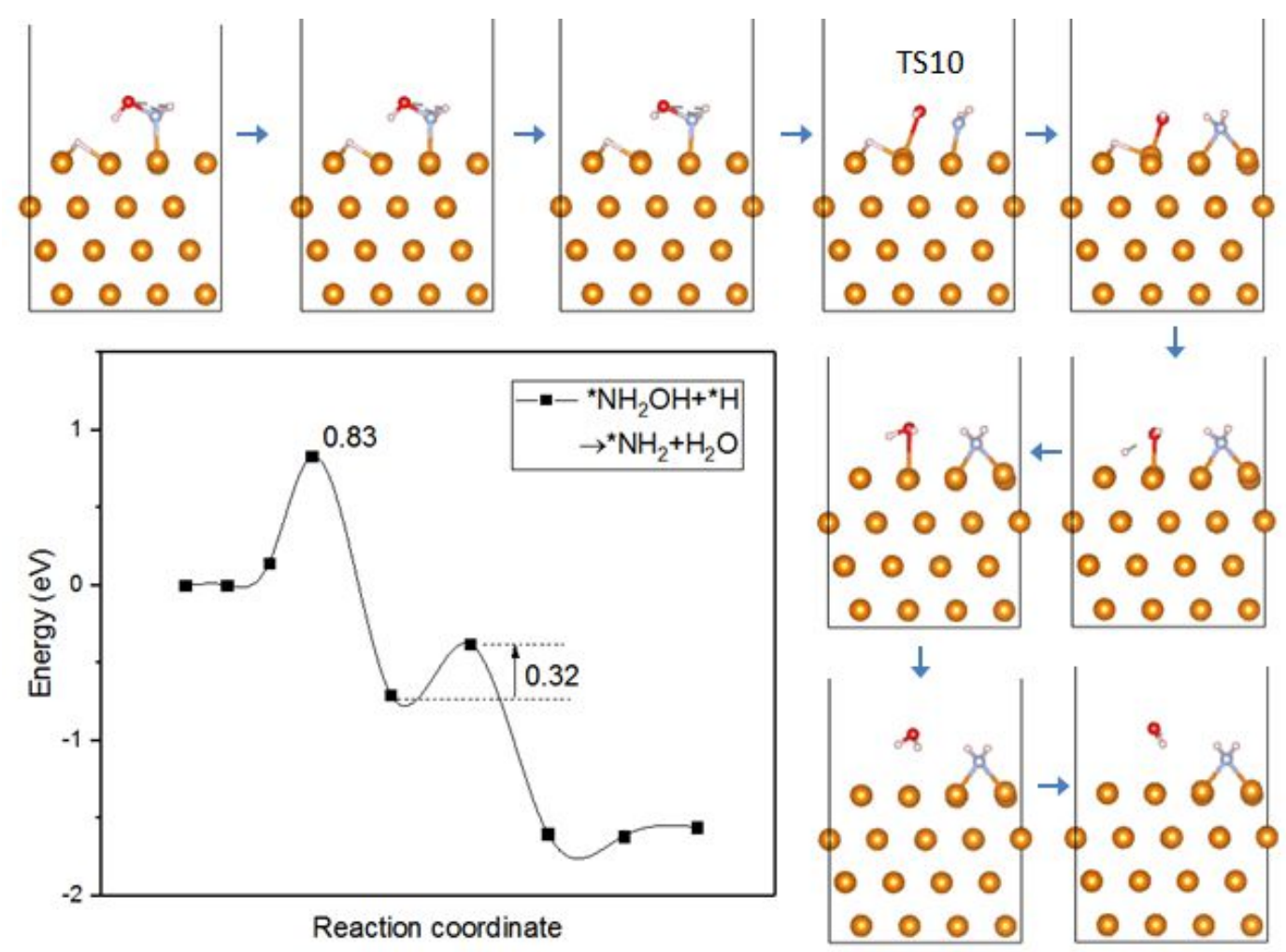

Figure S12. Minimum energy path and activation barrier of elementary step $* \mathrm{NH}_{2} \mathrm{OH}+* \mathrm{H} \rightarrow * \mathrm{NH}_{2}+\mathrm{H}_{2} \mathrm{O}$. TS10 stands for the transition state. 


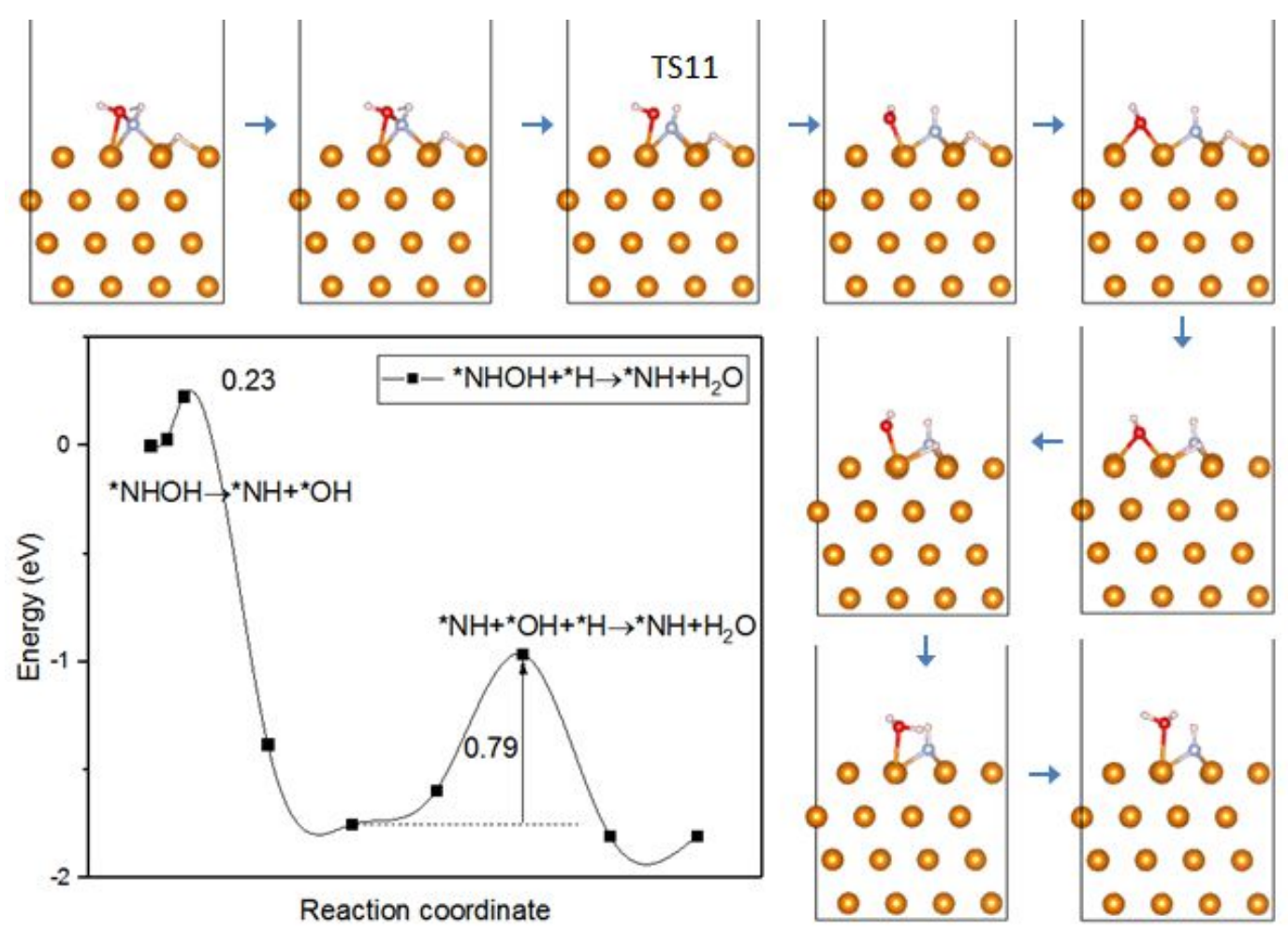

Figure S13. Minimum energy path and activation barrier of elementary step *NHOH$+* \mathrm{H} \rightarrow * \mathrm{NH}+\mathrm{H}_{2} \mathrm{O}$. TS11 stands for the transition state of breaking the N-O bond.

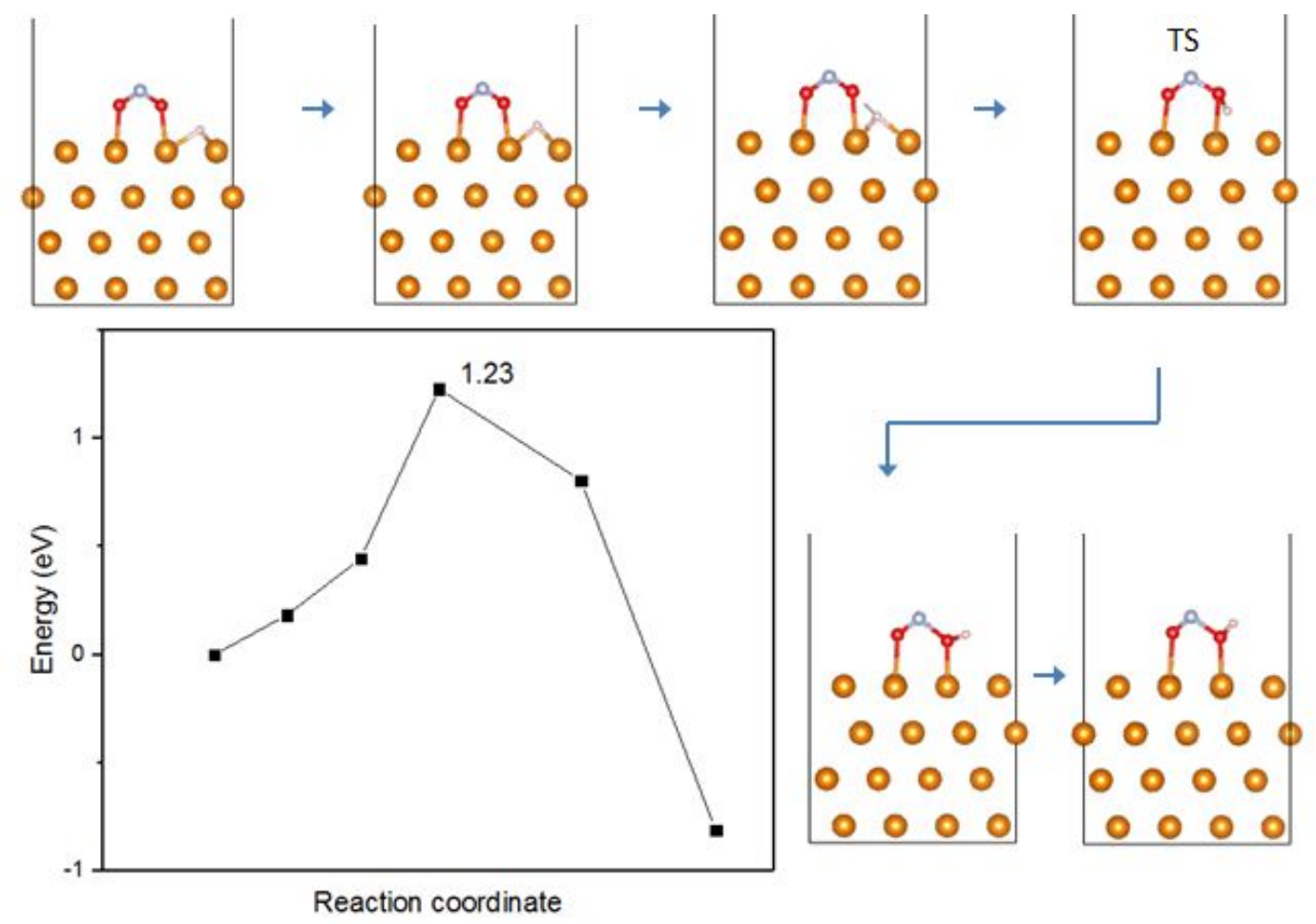

Figure S14. Minimum energy path and activation barrier of elementary step $* \mathrm{NO}_{2}+* \mathrm{H} \rightarrow{ }^{*} \mathrm{NO}_{2} \mathrm{H}$. TS stands for the transition state. 


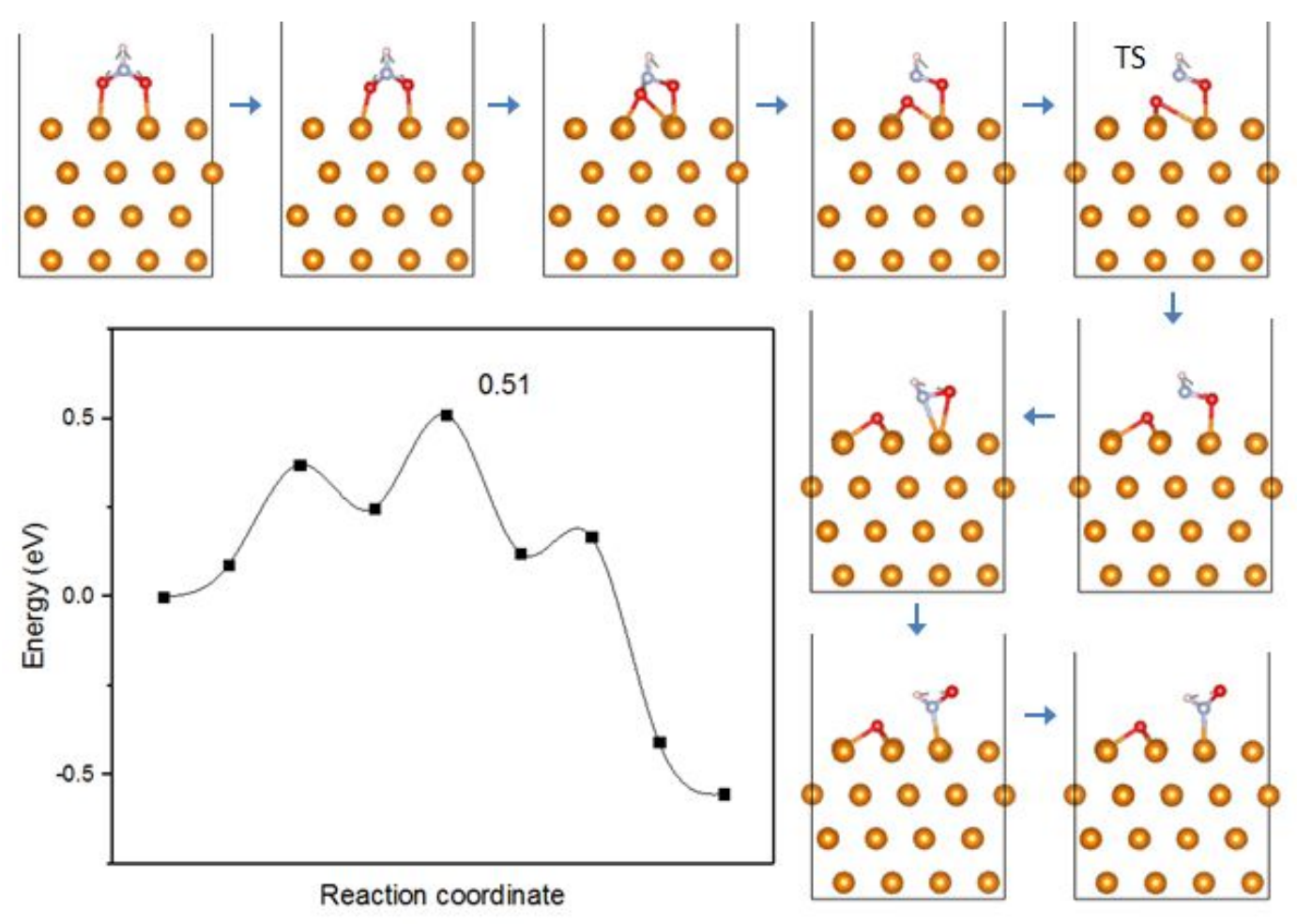

Figure S15. Minimum energy path and activation barrier of elementary step $* \mathrm{NO}_{2} \mathrm{H} \rightarrow * \mathrm{NHO}+* \mathrm{O}$. TS stands for the transition state.

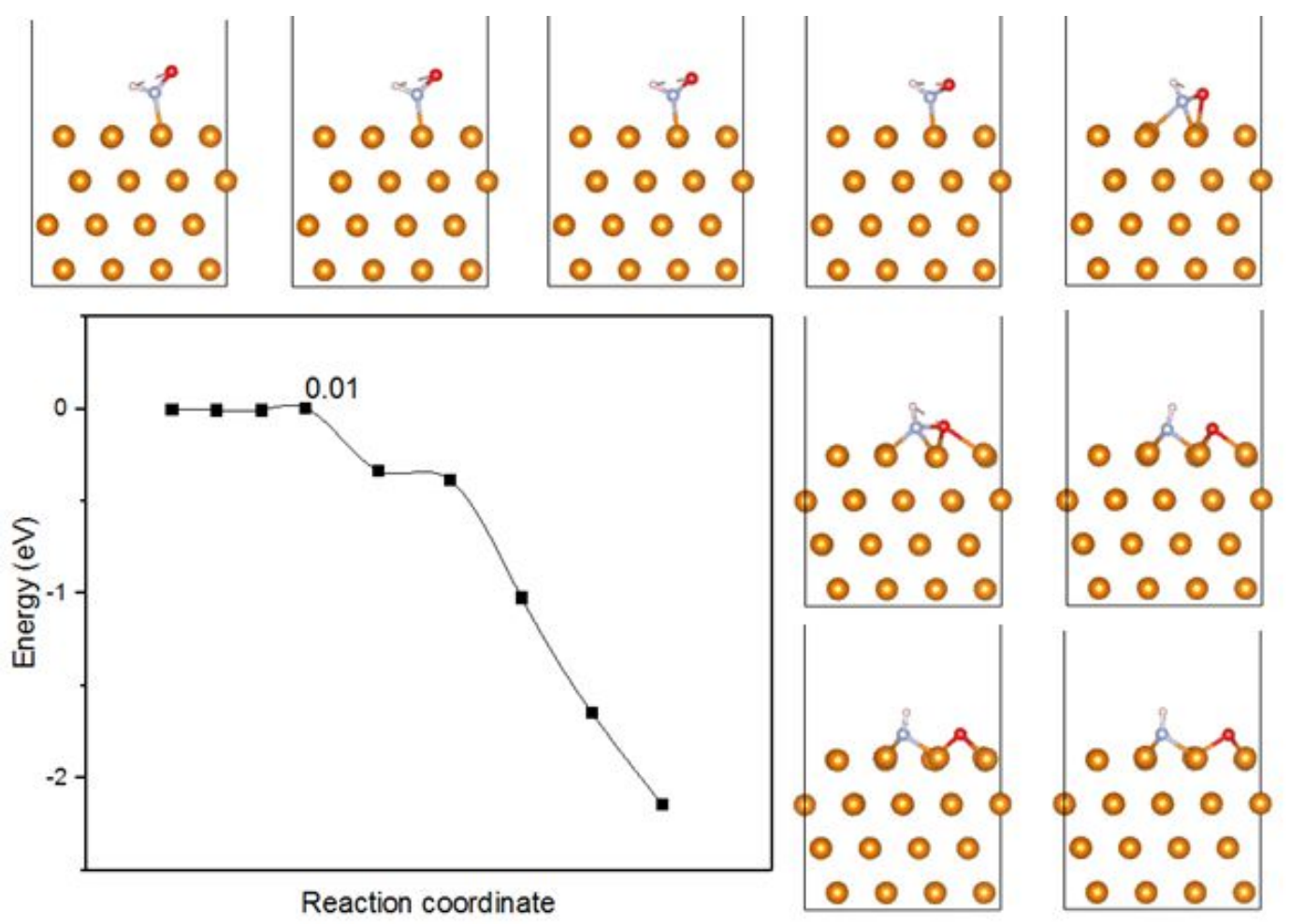

Figure S16. Minimum energy path and activation barrier of elementary step $* \mathrm{NHO} \rightarrow{ }^{*} \mathrm{NH}+* \mathrm{O}$. TS stands for the transition state. 


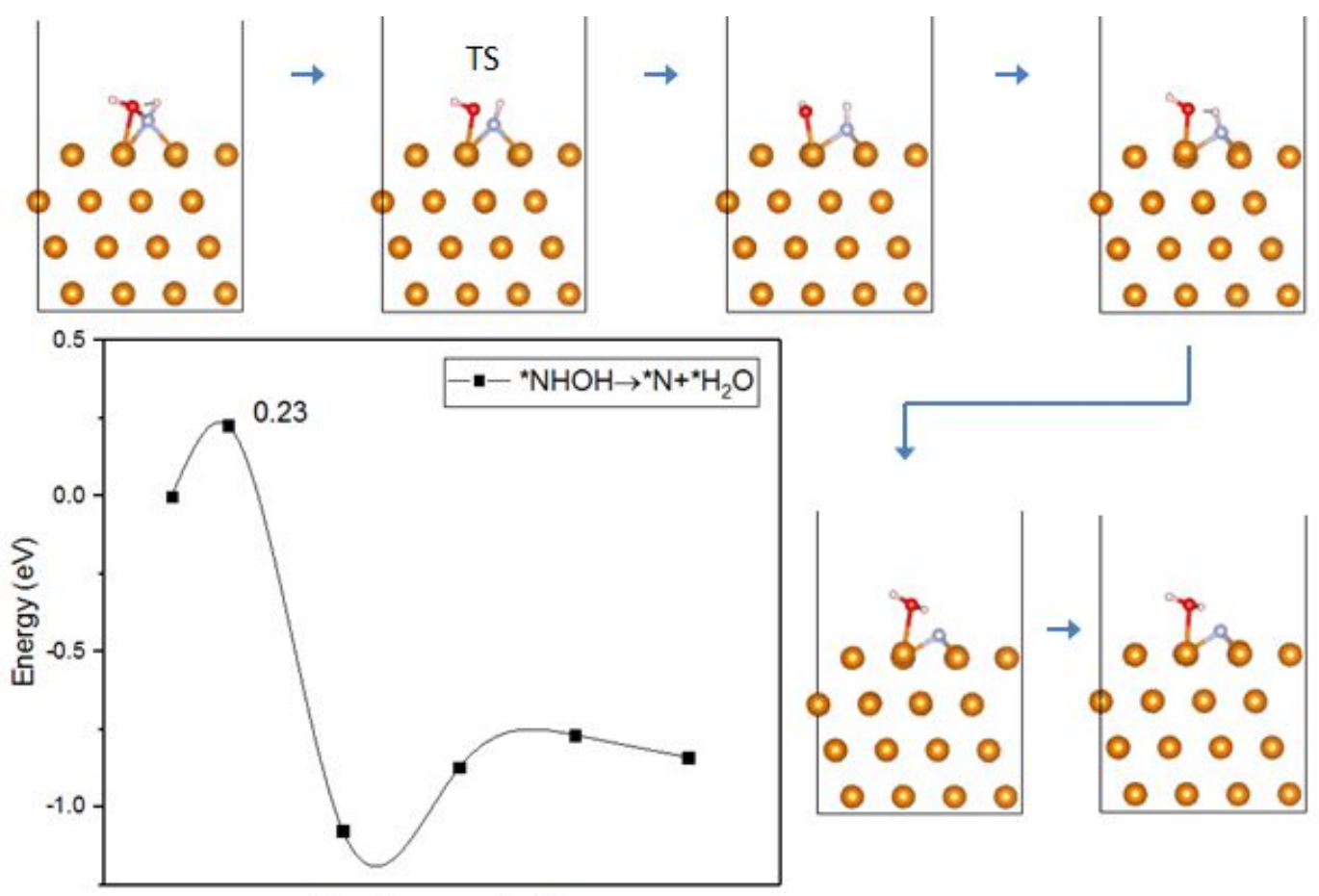

Reaction coordinate

Figure S17. Minimum energy path and activation barrier of elementary step *NHOH $\rightarrow{ }^{*} \mathrm{~N}+* \mathrm{H}_{2} \mathrm{O}$.

TS stands for the transition state.

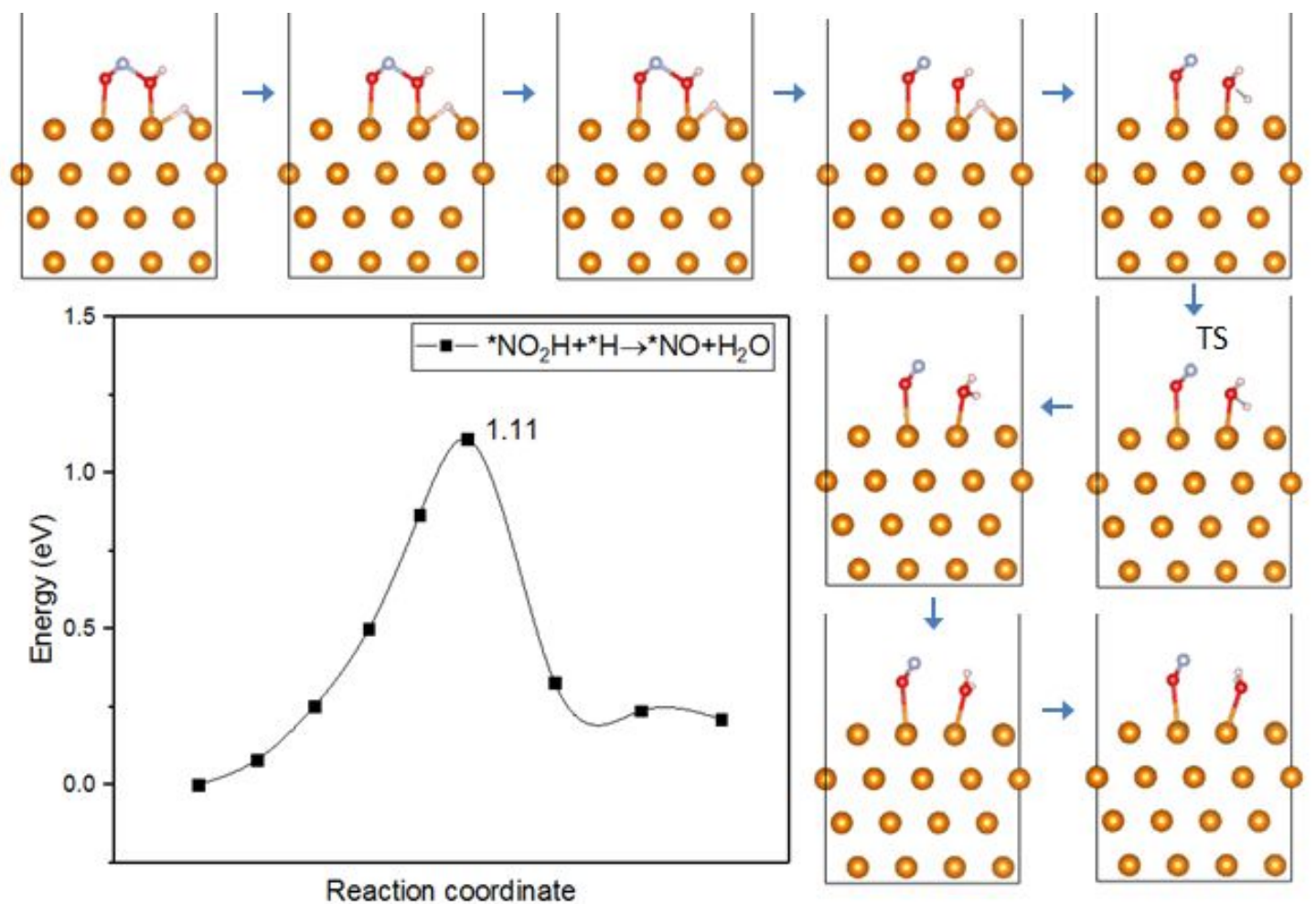

Figure S18. Minimum energy path and activation barrier of elementary step $* \mathrm{NO}_{2} \mathrm{H}+* \mathrm{H} \rightarrow * \mathrm{NO}+\mathrm{H}_{2} \mathrm{O}$. TS stands for the transition state. 


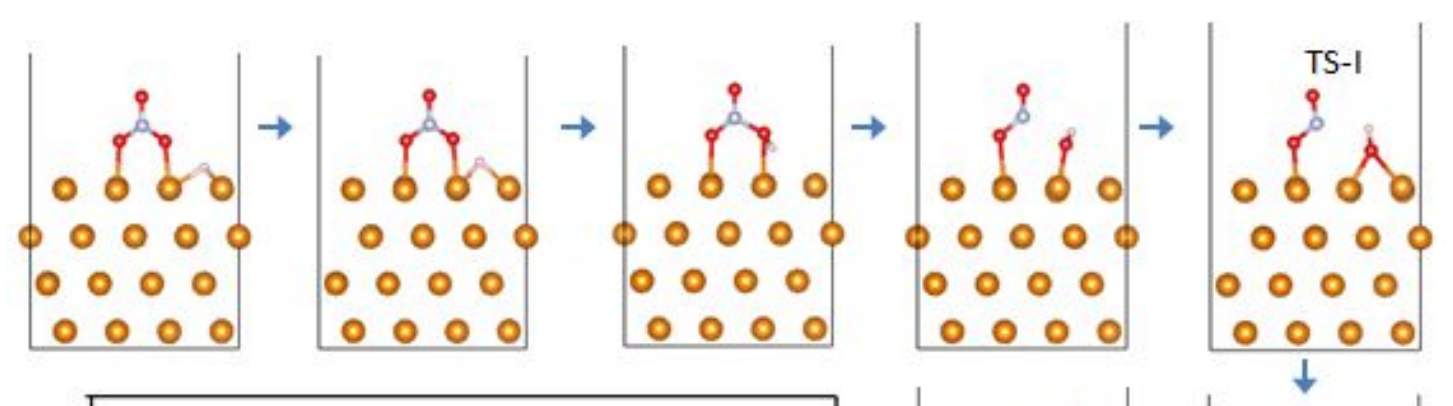

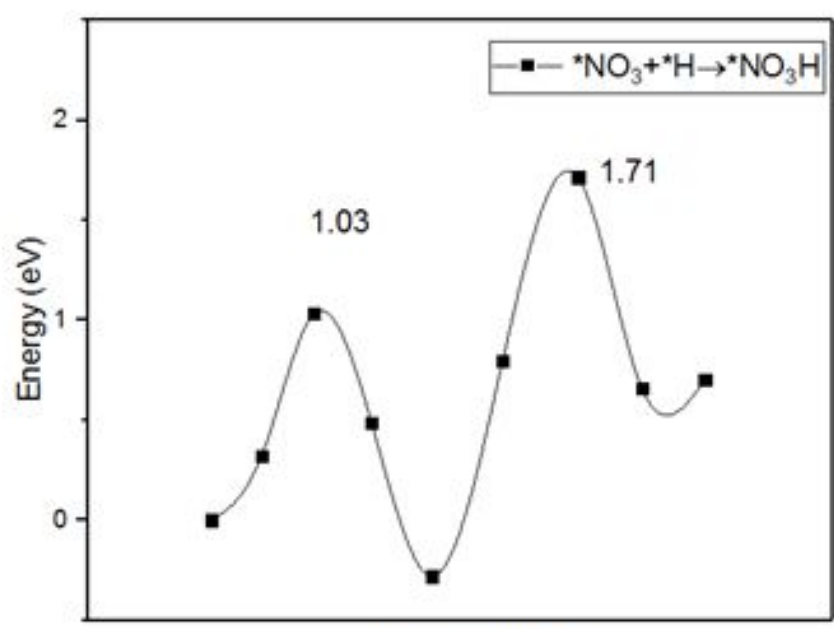

Reaction coordinate

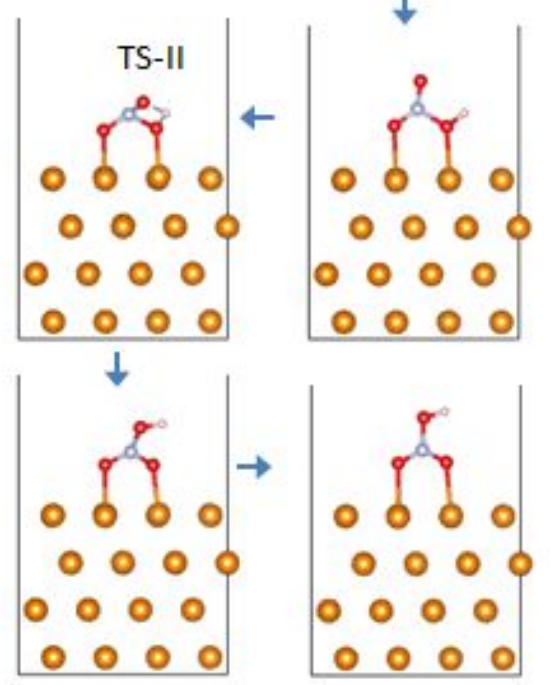

Figure S19. Minimum energy path and activation barrier of elementary step $* \mathrm{NO}_{3}+* \mathrm{H} \rightarrow{ }^{*} \mathrm{NO}_{3} \mathrm{H}$. TS stands for the transition state.
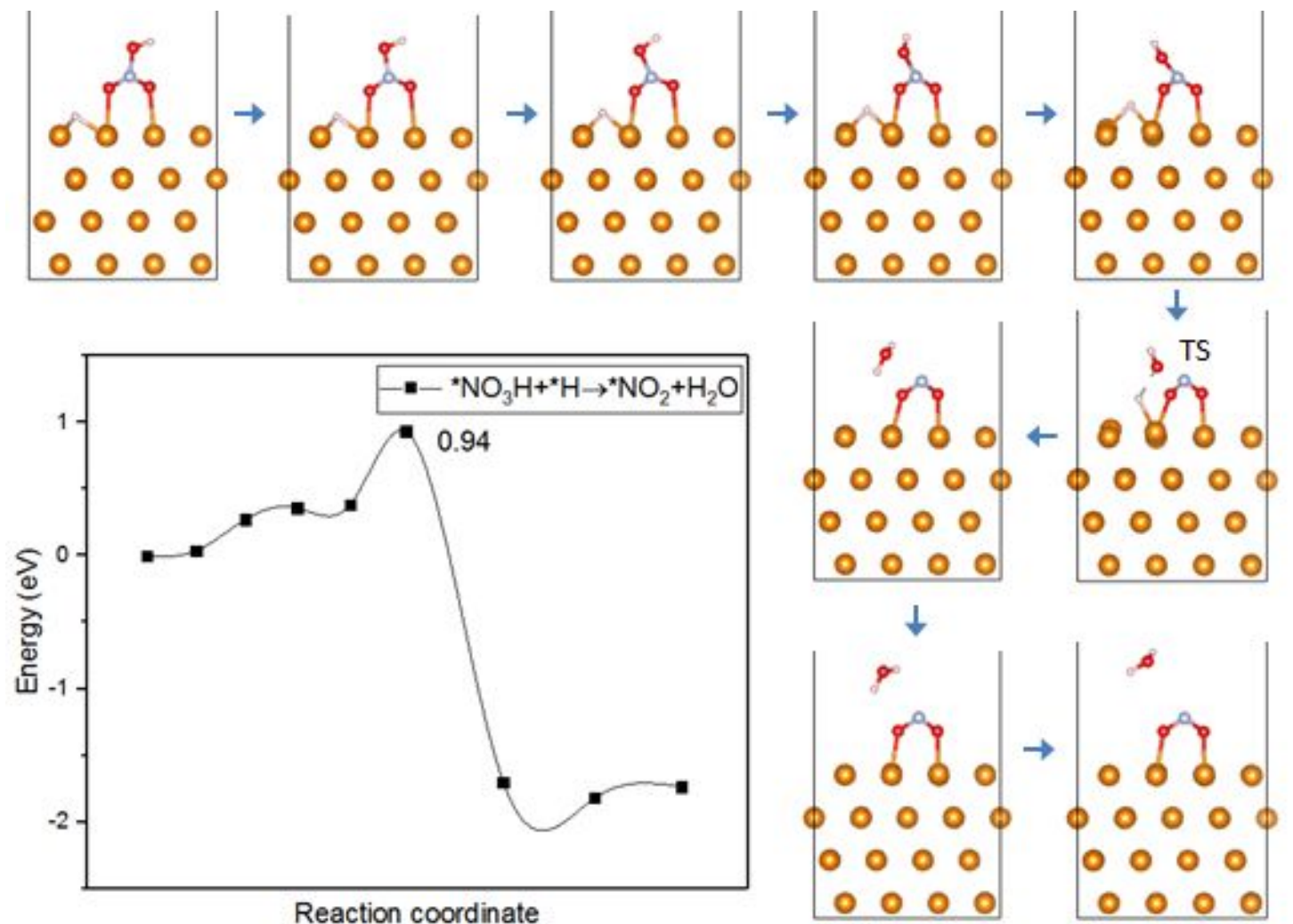

Figure S20. Minimum energy path and activation barrier of elementary step 
$* \mathrm{NO}_{3} \mathrm{H}+* \mathrm{H} \rightarrow * \mathrm{NO}_{2}+\mathrm{H}_{2} \mathrm{O}$. TS stands for the transition state.

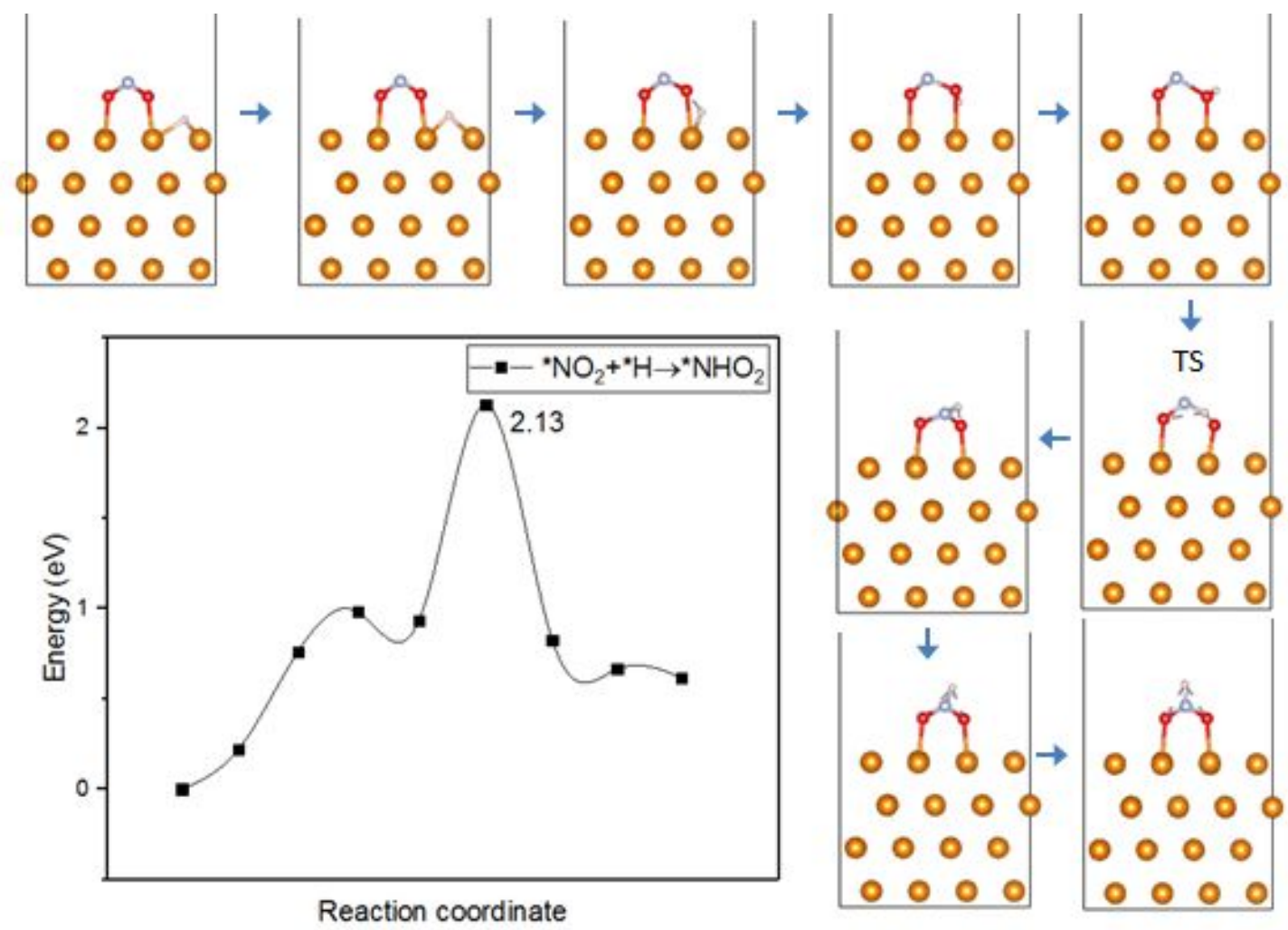

Figure S21. Minimum energy path and activation barrier of elementary step $* \mathrm{NO}_{2}+* \mathrm{H} \rightarrow * \mathrm{NHO}_{2}$.

TS stands for the transition state.

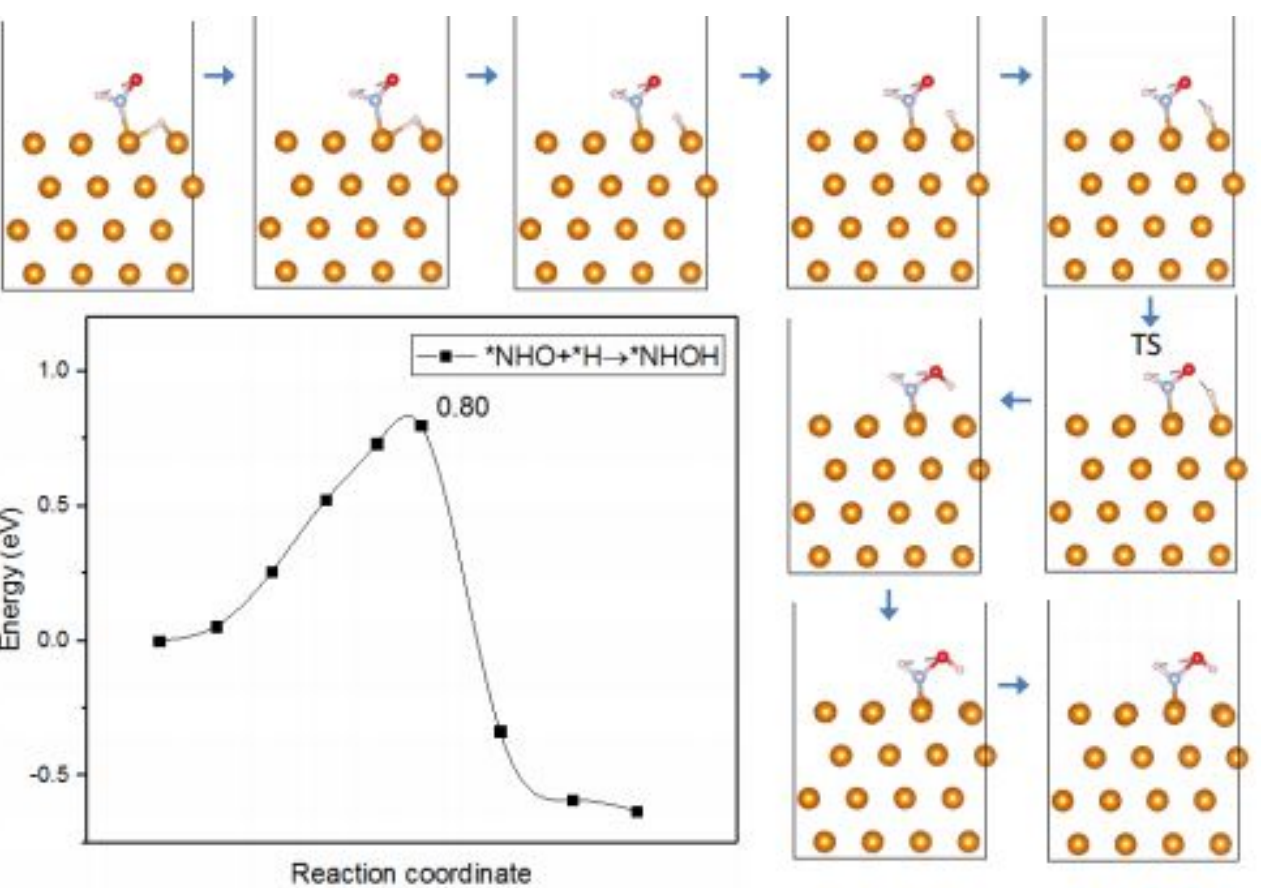

Figure S22. Minimum energy path and activation barrier of elementary step $* \mathrm{NHO}+{ }^{*} \mathrm{H} \rightarrow * \mathrm{NHOH}$. TS stands for the transition state. 

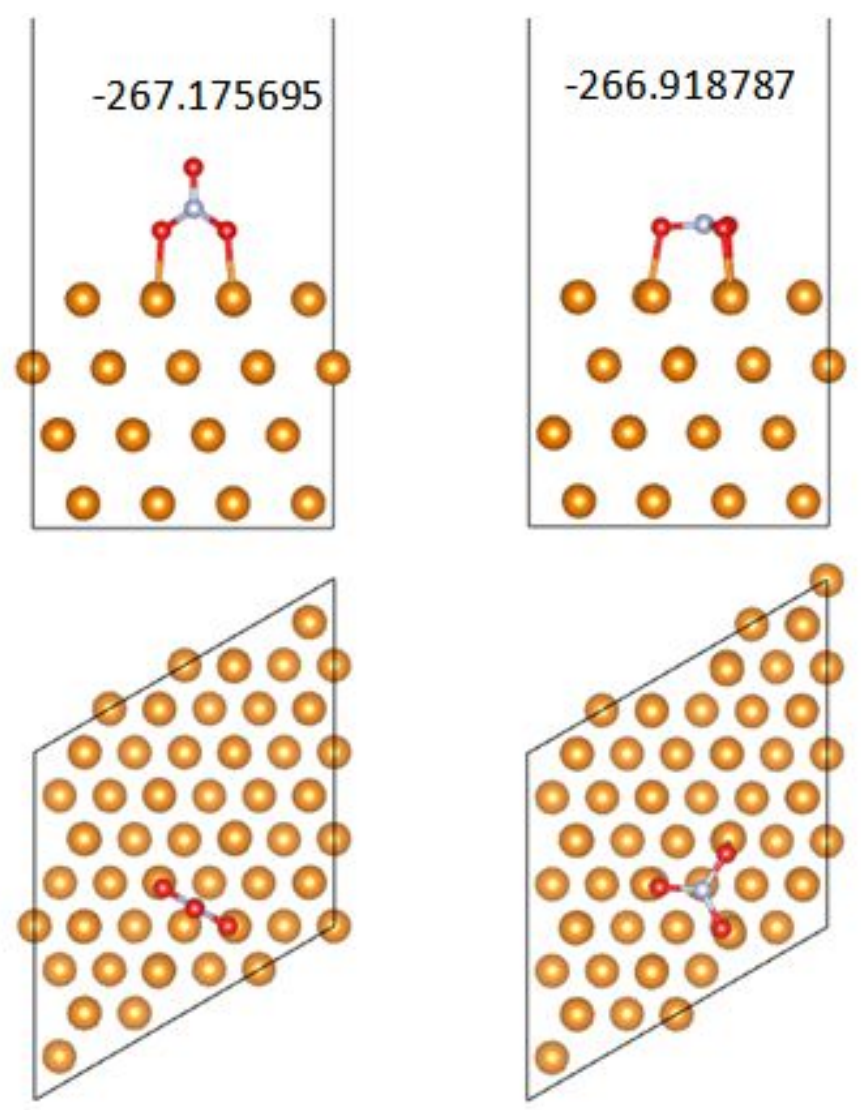

Figure S23. Adsorption mode of $* \mathrm{NO}_{3}$. The O, O-bidentate configuration is thermodynamically more stable than the palallel adsorption configuration.

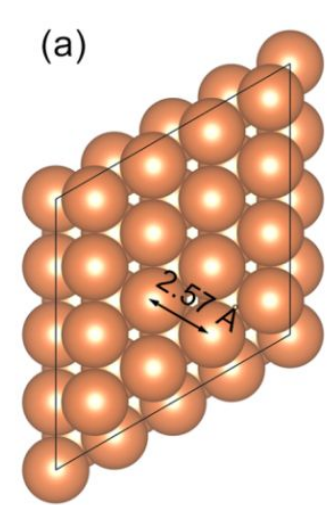

(b)

(c)

(g)
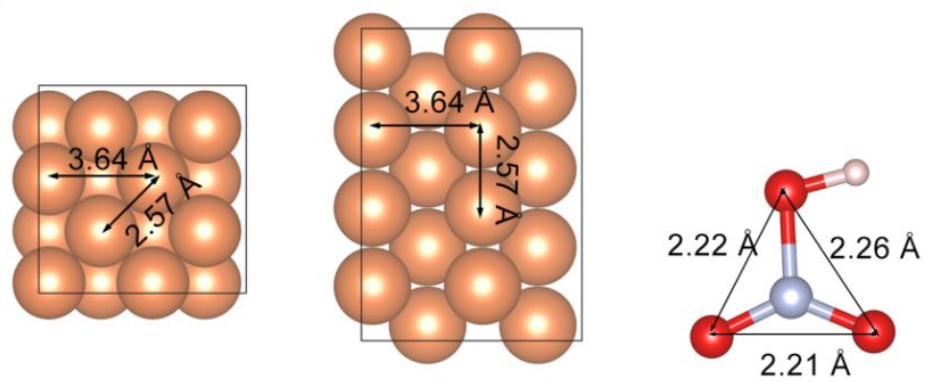

(d)

(e)

(f)
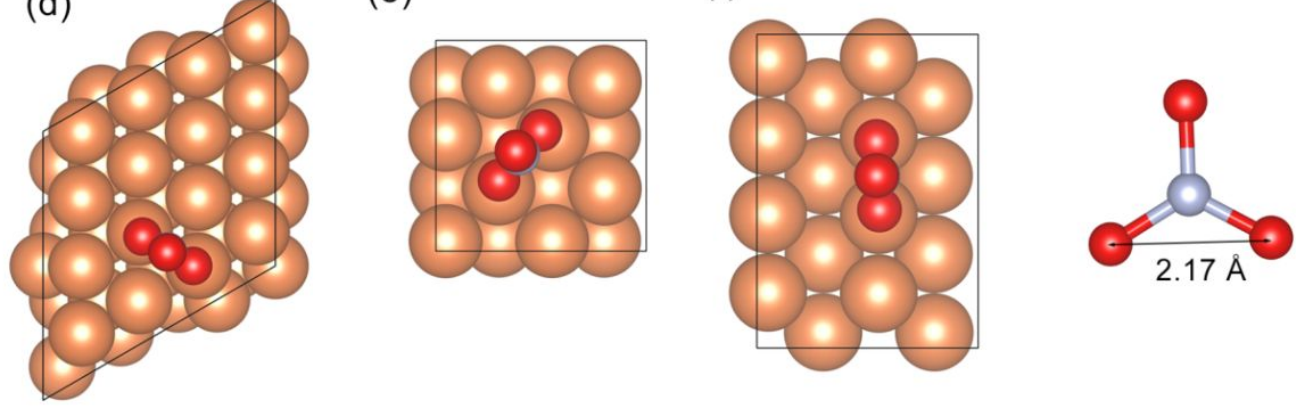

Figure S24. The nitrate adsorption prefers the stereospecific $\mathrm{Cu}-\mathrm{Cu}$ geometry that matches the O-O distance in $\mathrm{NO}_{3}{ }^{-}$and $\mathrm{HNO}_{3}$. Optimized structrures of (a) $\mathrm{Cu}$ (111) (b) $\mathrm{Cu}$ (100) (c) $\mathrm{Cu}(110)$ 
and optimized $* \mathrm{NO}_{3}$ adsorption configurations on $\mathrm{Cu}$ surfaces (d) $* \mathrm{NO}_{3} @ \mathrm{Cu}$ (111), (e) *NO 3 @ $\mathrm{Cu}(100)$, (f) *NO $3 @ \mathrm{Cu}(110)$. (g) Optimized structures of $\mathrm{HNO}_{3}$ and $\mathrm{NO}_{3}{ }^{-}$.

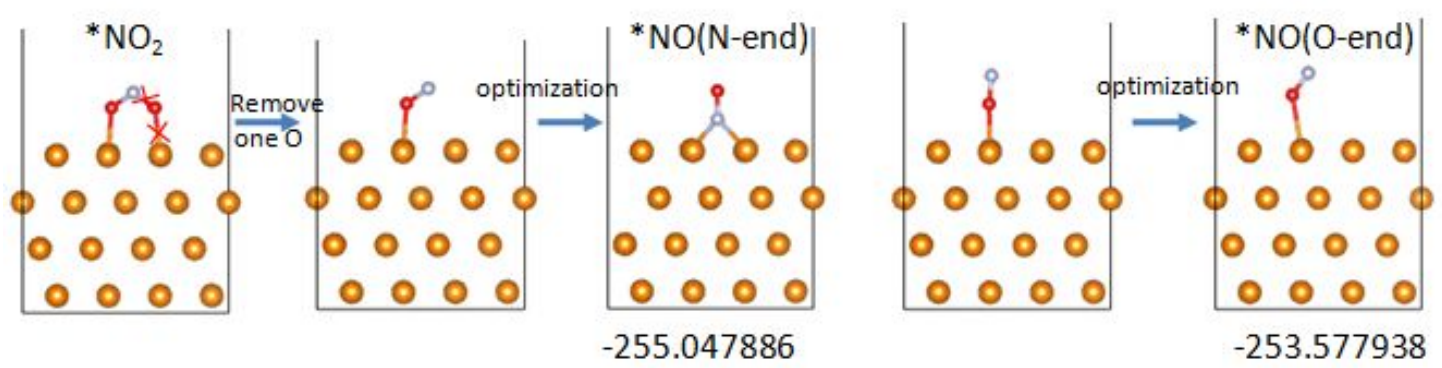

Figure S25. Comparison of $* \mathrm{NO}\left(\mathrm{N}\right.$-end) and $* \mathrm{NO}(\mathrm{O}-\mathrm{end}) .{ }^{*} \mathrm{NO}(\mathrm{N}$-end) is more stable than *NO(O-end).

Table S14. Calculated $\mathrm{U}^{0}$ by the method developed by Janik et al. ${ }^{4}$.

\begin{tabular}{|c|c|c|c|c|c|c|}
\hline & Reaction & Reference equation for $\mathrm{U}^{0}$ & $\Delta \mathrm{E}^{\mathrm{a}}$ & $\mathrm{T} \Delta \mathrm{S}$ & $\triangle \mathrm{ZPE}$ & $\mathrm{U}^{0 \mathrm{~b}}$ \\
\hline $\mathrm{TS}^{\mathrm{c}}$ & $* \mathrm{NO}_{3} \rightarrow * \mathrm{NO}_{2}+* \mathrm{O}$ & - & - & - & - & - \\
\hline TS2 & $* \mathrm{NO}_{2} \rightarrow * \mathrm{NO}+* \mathrm{O}$ & - & - & - & - & - \\
\hline TS3 & $* \mathrm{NO} \rightarrow * \mathrm{~N}+* \mathrm{O}$ & - & - & - & - & - \\
\hline TS4 & $* \mathrm{~N}+* \mathrm{H} \rightarrow * \mathrm{NH}$ & $* \mathrm{~N}+\mathrm{H}^{+}+\mathrm{e}^{-} \rightarrow * \mathrm{~N}+* \mathrm{H}$ & -0.209 & 0.205 & 0.045 & -0.041 \\
\hline TS5 & $* \mathrm{NH}+* \mathrm{H} \rightarrow * \mathrm{NH}_{2}$ & $* \mathrm{NH}+\mathrm{H}^{+}+\mathrm{e}^{-} \rightarrow * \mathrm{NH}+* \mathrm{H}$ & -0.242 & 0.205 & 0.035 & 0.002 \\
\hline TS6 & $* \mathrm{NH}_{2}+* \mathrm{H} \rightarrow * \mathrm{NH}_{3}$ & $* \mathrm{NH}_{2}+\mathrm{H}^{+}+\mathrm{e}^{-} \rightarrow * \mathrm{NH}_{2}+* \mathrm{H}$ & -0.288 & 0.205 & 0.035 & 0.048 \\
\hline TS7 & $* \mathrm{NO}+* \mathrm{H} \rightarrow * \mathrm{NOH}$ & $* \mathrm{NO}+\mathrm{H}^{+}+\mathrm{e}^{-} \rightarrow * \mathrm{NO}+* \mathrm{H}$ & -0.265 & 0.205 & 0.03 & 0.030 \\
\hline TS8 & $* \mathrm{NOH}+* \mathrm{H} \rightarrow * \mathrm{NHOH}$ & $* \mathrm{NOH}+\mathrm{H}^{+}+\mathrm{e}^{-} \rightarrow * \mathrm{NOH}+* \mathrm{H}$ & -0.269 & 0.205 & 0.055 & 0.009 \\
\hline TS9 & $* \mathrm{NHOH}+* \mathrm{H} \rightarrow * \mathrm{NH}_{2} \mathrm{OH}$ & $* \mathrm{NHOH}+\mathrm{H}^{+}+\mathrm{e}^{-} \rightarrow * \mathrm{NHOH}^{+*} \mathrm{H}$ & -0.313 & 0.205 & 0.035 & 0.073 \\
\hline TS10 & $* \mathrm{NH}_{2} \mathrm{OH}+* \mathrm{H} \rightarrow * \mathrm{NH}_{2}+\mathrm{H}_{2} \mathrm{O}$ & $* \mathrm{NH}_{2} \mathrm{OH}+\mathrm{H}^{+}+\mathrm{e}^{-} \rightarrow * \mathrm{NH}_{2} \mathrm{OH}+* \mathrm{H}$ & -0.443 & 0.205 & 0.025 & 0.213 \\
\hline TS11 & $* \mathrm{NHOH}+* \mathrm{H} \rightarrow * \mathrm{NH}+\mathrm{H}_{2} \mathrm{O}$ & $* \mathrm{NHOH}+\mathrm{H}^{+}+\mathrm{e}^{-} \rightarrow * \mathrm{NHOH}+* \mathrm{H}$ & -0.313 & 0.205 & 0.035 & 0.073 \\
\hline
\end{tabular}

${ }^{\text {a }} \Delta \mathrm{E}=\mathrm{E}(* \mathrm{~A}+* \mathrm{H})-\mathrm{E}(* \mathrm{~A})-1 / 2 \mathrm{E}\left(\mathrm{H}_{2}\right)$

${ }^{b} \mathrm{U}^{0}$ is the potential where reaction $* \mathrm{~A}+\mathrm{H}^{+}+\mathrm{e}^{-} \rightarrow{ }^{*} \mathrm{~A}+* \mathrm{H}$ possess zero free energy change i.e. $\mathrm{G}\left({ }^{*} \mathrm{~A}+* \mathrm{H}\right)+\mathrm{eU}^{0}-\mathrm{G}\left({ }^{*} \mathrm{~A}\right)-1 / 2 \mathrm{G}\left(\mathrm{H}_{2}\right)=0$, where *A means the system with A adsorbed on the surface. For sake of calculation, $\mathrm{U}^{0}$ is written as $\mathrm{U}^{0}=-\left[\mathrm{G}\left({ }^{*} \mathrm{~A}+* \mathrm{H}\right)-\mathrm{G}\left({ }^{*} \mathrm{~A}\right)-1 / 2 \mathrm{G}\left(\mathrm{H}_{2}\right)\right] / \mathrm{e}=-[\Delta \mathrm{E}-\mathrm{T} \Delta \mathrm{S}+\Delta \mathrm{ZPE}] / \mathrm{e}$.

${ }^{c}$ TS1-TS3 are deoxygenation reactions. 
Table S15. Calculated $\mathrm{U}^{0}$ by the method used in Zhang et al.' work. ${ }^{5}$

\begin{tabular}{|c|c|c|c|c|}
\hline & Reaction & $\mathrm{Ea}\left(\mathrm{U}^{0}\right)(\mathrm{eV})$ & work function $(\mathrm{eV})$ & $\mathrm{U}^{0}(\mathrm{~V})$ \\
\hline TS1 & $* \mathrm{NO}_{3} \rightarrow * \mathrm{NO}_{2}+* \mathrm{O}$ & 0.68 & 4.78 & 0.35 \\
\hline TS2 & $* \mathrm{NO}_{2} \rightarrow * \mathrm{NO}+* \mathrm{O}$ & 0.98 & 4.64 & 0.21 \\
\hline TS3 & $* \mathrm{NO} \rightarrow * \mathrm{~N}+* \mathrm{O}$ & 1.63 & 4.46 & 0.03 \\
\hline TS4 & $* \mathrm{~N}+* \mathrm{H} \rightarrow * \mathrm{NH}$ & 0.67 & 4.44 & 0.01 \\
\hline TS5 & $* \mathrm{NH}+* \mathrm{H} \rightarrow * \mathrm{NH}_{2}$ & 0.84 & 4.46 & 0.03 \\
\hline TS6 & $* \mathrm{NH}_{2}+* \mathrm{H} \rightarrow * \mathrm{NH}_{3}$ & 1.16 & 4.48 & 0.05 \\
\hline TS7 & $* \mathrm{NO}+* \mathrm{H} \rightarrow * \mathrm{NOH}$ & 0.98 & 4.46 & 0.03 \\
\hline TS8 & $* \mathrm{NOH}+* \mathrm{H} \rightarrow * \mathrm{NHOH}$ & 0.85 & 4.41 & -0.02 \\
\hline TS9 & $* \mathrm{NHOH}+* \mathrm{H} \rightarrow * \mathrm{NH}_{2} \mathrm{OH}$ & 1.36 & 4.52 & 0.09 \\
\hline TS10 & $* \mathrm{NH}_{2} \mathrm{OH}+* \mathrm{H} \rightarrow * \mathrm{NH}_{2}+\mathrm{H}_{2} \mathrm{O}$ & 0.83 & 4.64 & 0.21 \\
\hline TS11 & $* \mathrm{NHOH}+* \mathrm{H} \rightarrow * \mathrm{NH}+\mathrm{H}_{2} \mathrm{O}$ & 0.23 & 4.52 & 0.09 \\
\hline
\end{tabular}

Table S16. Comparison of $E_{a}(U)$ calculated by two methods a

\begin{tabular}{|c|c|c|c|c|}
\hline & Reaction & $\mathrm{E}_{\mathrm{a}}\left(\mathrm{U}^{0}\right)$ & $\mathrm{E}_{\mathrm{a}}(\mathrm{U})$-Method 1 & $\mathrm{E}_{\mathrm{a}}(\mathrm{U})-$ Method 2 \\
\hline TS1 & $* \mathrm{NO}_{3} \rightarrow * \mathrm{NO}_{2}+* \mathrm{O}$ & 0.68 & - & $0.68+1 *(\mathrm{U}-0.35)$ \\
\hline TS2 & $* \mathrm{NO}_{2} \rightarrow * \mathrm{NO}+* \mathrm{O}$ & 0.98 & - & $0.98+1 *(\mathrm{U}-0.21)$ \\
\hline TS3 & $* \mathrm{NO} \rightarrow{ }^{*} \mathrm{~N}+{ }^{*} \mathrm{O}$ & 1.63 & - & $1.63+1 *(\mathrm{U}-0.03)$ \\
\hline TS4 & $* \mathrm{~N}+* \mathrm{H} \rightarrow * \mathrm{NH}$ & 0.67 & $0.67+0.5^{*}(\mathrm{U}+0.041)$ & $0.67+0.5 *(\mathrm{U}-0.01)$ \\
\hline TS5 & $* \mathrm{NH}+* \mathrm{H} \rightarrow * \mathrm{NH}_{2}$ & 0.84 & $0.84+0.5^{*}(\mathrm{U}-0.002)$ & $0.84+0.5^{*}(\mathrm{U}-0.03)$ \\
\hline TS6 & $* \mathrm{NH}_{2}+* \mathrm{H} \rightarrow * \mathrm{NH}_{3}$ & 1.16 & $1.16+0.5 *(\mathrm{U}-0.048)$ & $1.16+0.5 *(\mathrm{U}-0.05)$ \\
\hline TS7 & $* \mathrm{NO}+* \mathrm{H} \rightarrow * \mathrm{NOH}$ & 0.98 & $0.98+0.5^{*}(\mathrm{U}-0.030)$ & $0.98+0.5 *(\mathrm{U}-0.03)$ \\
\hline TS8 & $* \mathrm{NOH}+* \mathrm{H} \rightarrow * \mathrm{NHOH}$ & 0.85 & $0.85+0.5^{*}(\mathrm{U}-0.009)$ & $0.85+0.5^{*}(\mathrm{U}+0.02)$ \\
\hline TS9 & $* \mathrm{NHOH}+* \mathrm{H} \rightarrow * \mathrm{NH}_{2} \mathrm{OH}$ & 1.36 & $1.36+0.5 *(\mathrm{U}-0.073)$ & $1.36+0.5 *(\mathrm{U}-0.09)$ \\
\hline TS10 & $* \mathrm{NH}_{2} \mathrm{OH}+* \mathrm{H} \rightarrow * \mathrm{NH}_{2}+\mathrm{H}_{2} \mathrm{O}$ & 0.83 & $0.83+0.5^{*}(\mathrm{U}-0.213)$ & $0.83+0.5 *(\mathrm{U}-0.21)$ \\
\hline TS11 & $* \mathrm{NHOH}+* \mathrm{H} \rightarrow * \mathrm{NH}+\mathrm{H}_{2} \mathrm{O}$ & 0.23 & $0.23+0.5 *(\mathrm{U}-0.073)$ & $0.23+0.5^{*}(\mathrm{U}-0.09)$ \\
\hline
\end{tabular}

${ }^{a}$ the results of hydrogenation reactions calculated by two methods give similar trends. 
Table S17. Influence of applied potential on $\Delta \mathrm{G}$ (in eV) of $\mathrm{NRA} 3$ on $\mathrm{Cu}(111)$ at $\mathrm{pH}=0$

\begin{tabular}{|c|c|c|c|}
\hline & Reaction & $\mathrm{U}=0 \mathrm{~V}$ & $\mathrm{U}($ in $\mathrm{V})$ \\
\hline$\Delta \mathrm{G} 1$ & $\mathrm{NO}_{3}^{-}(1)+* \rightarrow * \mathrm{NO}_{3}+\mathrm{e}^{-}$ & 0.06 & $0.06-\mathrm{U}^{\text {a }}$ \\
\hline$\Delta \mathrm{G} 2$ & $* \mathrm{NO}_{3}+2 \mathrm{H}^{+}+2 \mathrm{e}^{-} \rightarrow * \mathrm{NO}_{2}+\mathrm{H}_{2} \mathrm{O}$ & -1.81 & $-1.81+2 * \mathrm{U}^{\mathrm{b}}$ \\
\hline$\Delta \mathrm{G} 3$ & $* \mathrm{NO}_{2}+2 \mathrm{H}^{+}+2 \mathrm{e}^{-} \rightarrow * \mathrm{NO}+\mathrm{H}_{2} \mathrm{O}$ & -1.16 & $-1.16+2 * U$ \\
\hline$\Delta \mathrm{G} 4$ & $* \mathrm{NO}+\mathrm{H}^{+}+\mathrm{e}^{-} \rightarrow * \mathrm{NOH}$ & 0.08 & $0.08+\mathrm{U}$ \\
\hline$\Delta \mathrm{G} 5$ & $* \mathrm{NOH}+\mathrm{H}^{+}+\mathrm{e}^{-} \rightarrow * \mathrm{NHOH}$ & -0.27 & $-0.27+U$ \\
\hline$\Delta \mathrm{G} 6$ & $* \mathrm{NHOH}+\mathrm{H}^{+}+\mathrm{e}^{-} \rightarrow * \mathrm{NH}+\mathrm{H}_{2} \mathrm{O}$ & -1.86 & $-1.86+U$ \\
\hline$\Delta \mathrm{G} 7$ & $* \mathrm{NH}+\mathrm{H}^{+}+\mathrm{e}^{-} \rightarrow * \mathrm{NH}_{2}$ & -0.42 & $-0.42+\mathrm{U}$ \\
\hline$\Delta \mathrm{G} 8$ & $* \mathrm{NH}_{2}+\mathrm{H}^{+}+\mathrm{e}^{-} \rightarrow * \mathrm{NH}_{3}$ & -0.53 & $-0.53+\mathrm{U}$ \\
\hline$\Delta \mathrm{G} 9$ & $* \mathrm{NH}_{3} \rightarrow *+\mathrm{NH}_{3}(\mathrm{~g})$ & 0.37 & 0.37 \\
\hline
\end{tabular}

Table S18. Influence of applied potential on $\Delta \mathrm{G}$ (in $\mathrm{eV}$ ) of $\mathrm{NRA} 3$ on $\mathrm{Cu}(111)$ at $\mathrm{pH}=14$

\begin{tabular}{|l|l|c|c|}
\hline & Reaction & $\mathrm{U}=0 \mathrm{~V}$ & $\mathrm{U}($ in V) \\
\hline$\Delta \mathrm{G} 1$ & $\mathrm{NO}_{3}^{-}(1)+* \rightarrow * \mathrm{NO}_{3}+\mathrm{e}^{-}$ & 0.06 & $0.06-\mathrm{U}^{\mathrm{a}}$ \\
\hline$\Delta \mathrm{G} 2$ & $* \mathrm{NO}_{3}+2 \mathrm{H}^{+}+2 \mathrm{e}^{-} \rightarrow * \mathrm{NO}_{2}+\mathrm{H}_{2} \mathrm{O}$ & -0.16 & $-0.16+2 * \mathrm{U}^{\mathrm{b}}$ \\
\hline$\Delta \mathrm{G} 3$ & $* \mathrm{NO}_{2}+2 \mathrm{H}^{+}+2 \mathrm{e}^{-} \rightarrow * \mathrm{NO}+\mathrm{H}_{2} \mathrm{O}$ & 0.49 & $0.49+2 * \mathrm{U}$ \\
\hline$\Delta \mathrm{G} 4$ & $* \mathrm{NO}+\mathrm{H}^{+}+\mathrm{e}^{-} \rightarrow * \mathrm{NOH}$ & 0.91 & $0.91+\mathrm{U}$ \\
\hline$\Delta \mathrm{G} 5$ & $* \mathrm{NOH}+\mathrm{H}^{+}+\mathrm{e}^{-} \rightarrow * \mathrm{NHOH}$ & 0.55 & $0.55+\mathrm{U}$ \\
\hline$\Delta \mathrm{G} 6$ & $* \mathrm{NHOH}+\mathrm{H}^{+}+\mathrm{e}^{-} \rightarrow * \mathrm{NH}+\mathrm{H}_{2} \mathrm{O}$ & -1.04 & $-1.04+\mathrm{U}$ \\
\hline$\Delta \mathrm{G} 7$ & $* \mathrm{NH}^{+} \mathrm{H}^{+}+\mathrm{e}^{-} \rightarrow * \mathrm{NH}_{2}$ & 0.40 & $0.40+\mathrm{U}$ \\
\hline$\Delta \mathrm{G} 8$ & $* \mathrm{NH}_{2}+\mathrm{H}^{+}+\mathrm{e}^{-} \rightarrow * \mathrm{NH}_{3}$ & 0.30 & $0.30+\mathrm{U}$ \\
\hline$\Delta \mathrm{G} 9$ & $* \mathrm{NH}_{3} \rightarrow *+\mathrm{NH}_{3}(\mathrm{~g})$ & 0.37 & 0.37 \\
\hline
\end{tabular}

a $\Delta \mathrm{G} 1$ increases with negative $\mathrm{U}$ values, indicating the adsorption of $\mathrm{NO}_{3}{ }^{-}$on the negatively biased electrode becomes increasingly unfavorable.

b $\Delta \mathrm{G} 2$ and $\Delta \mathrm{G} 3$ involves two electrons transfer process, $\Delta \mathrm{G} 3-\Delta \mathrm{G} 9$ involve one electron transfer respectively. Negative $U$ values can substantially reduce the values of $\Delta \mathrm{G} 2-\Delta \mathrm{G}$. 

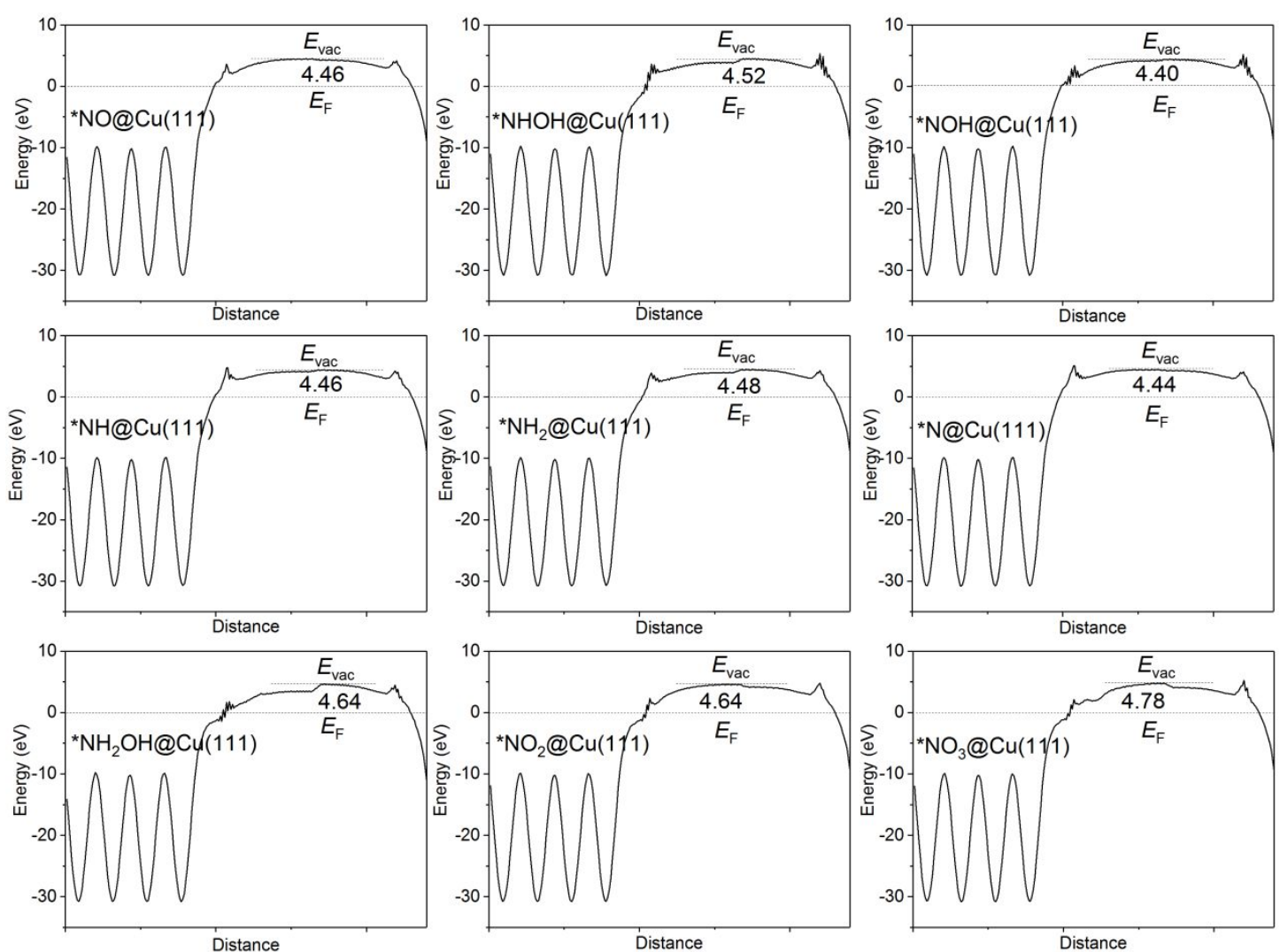

Figure S26. Workfunctions of $\mathrm{Cu}$ (111) with intermediates adsorbed.

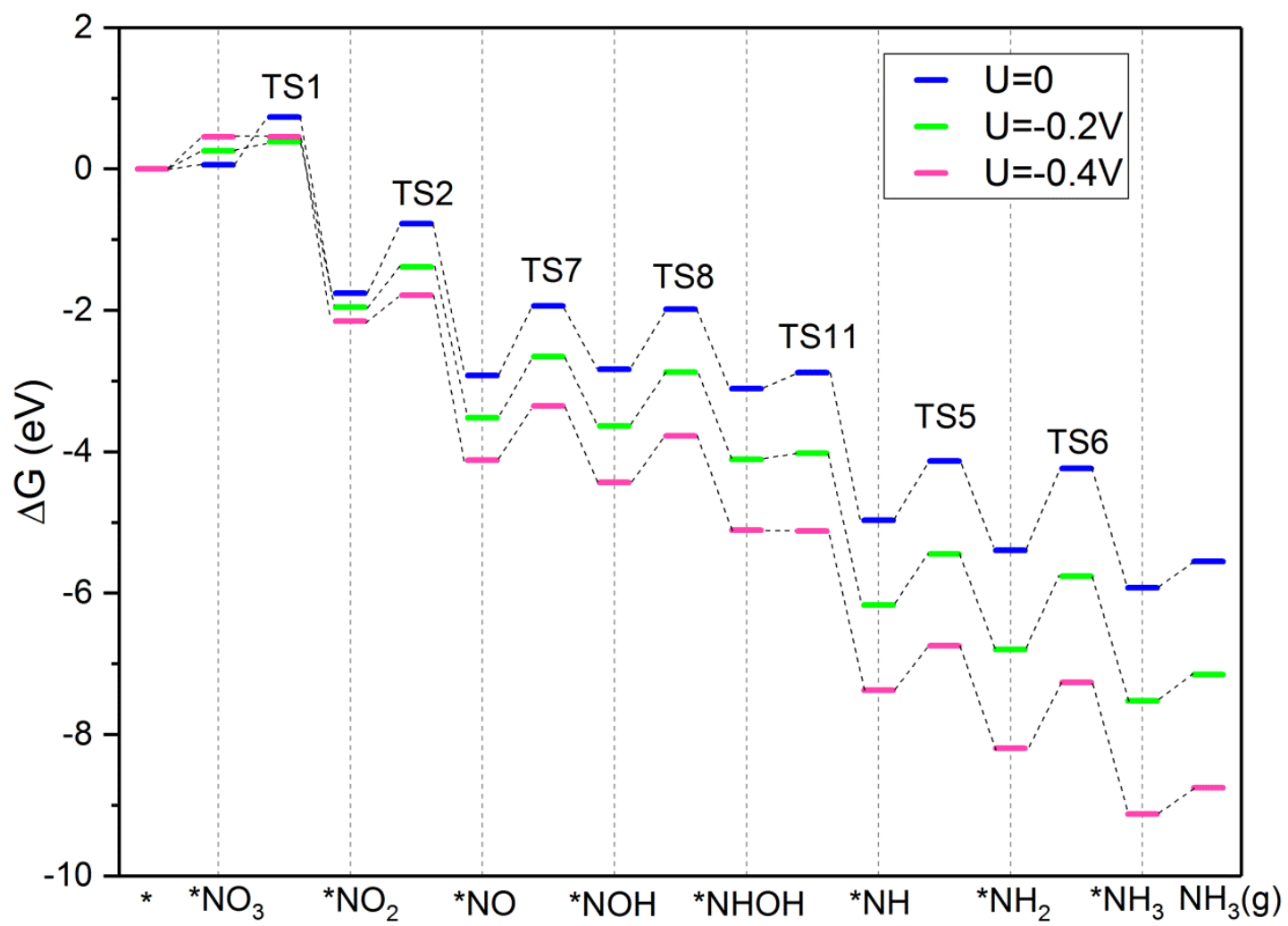

Figure S27. Preferred pathway NRA3 and associated reaction energetics identifified for production of $\mathrm{NH}_{3}$ starting with $\mathrm{NO}_{3}$ on $\mathrm{Cu}(111)$ surface at $0,-0.2 \mathrm{~V}$ and $-0.4 \mathrm{~V}$, vs. RHE. 


\section{References}

1. Valdes, A.; Qu, Z. W.; Kroes, G. J.; Rossmeisl, J.; Norskov, J. K. Oxidation and Photo-oxidation of Water on $\mathrm{TiO}_{2}$ Surface. J. Phys. Chem. C 2008, 112, 9872-9879.

2. Dean, J. A., Lange's Handbook of Chemistry (16th Edition). McGraw-Hill: New York, 1999.

3. Liu, J.-X.; Richards, D.; Singh, N.; Goldsmith, B. R. Activity and Selectivity Trends in Electrocatalytic Nitrate Reduction on Transition Metals. ACS Catal. 2019, 9, 7052-7064.

4. Akhade, S. A.; Bernstein, N. J.; Esopi, M. R.; Regula, M. J.; Janik, M. J. A Simple Method to Approximate Electrode Potential-dependent Activation Energies Using Density Functional Theory. Catal. Today 2017, 288, 63-73.

5. Guo, Y.; Cai, X.; Shen, S.; Wang, G.; Zhang, J. Computational Prediction and Experimental Evaluation of Nitrate Reduction to Ammonia on Rhodium. J. Catal. 2021, 402, 1-9. 